\title{
A high throughput robot system for machine vision based plant phenotype studies
}

\author{
Ram Subramanian • Edgar P. Spalding • \\ Nicola J. Ferrier
}

Received: 16 June 2011 / Revised: 8 April 2012 / Accepted: 2 May 2012 / Published online: 16 June 2012

(c) The Author(s) 2012. This article is published with open access at Springerlink.com

\begin{abstract}
This work demonstrates how a high throughput robotic machine vision systems can quantify seedling development with high spatial and temporal resolution. The throughput that the system provides is high enough to match the needs of functional genomics research. Analyzing images of plant seedlings growing and responding to stimuli is a proven approach to finding the effects of an affected gene. However, with $10^{4}$ genes in a typical plant genome, comprehensive studies will require high throughput methodologies. To increase throughput without sacrificing spatial or temporal resolution, a 3 axis robotic gantry system utilizing visual servoing was developed. The gantry consists of direct drive linear servo motors that can move the cameras at a speed of $1 \mathrm{~m} / \mathrm{s}$ with an accuracy of $1 \mu \mathrm{m}$, and a repeatability of $0.1 \mu \mathrm{m}$. Perpendicular to the optical axis of the cameras was a $1 \mathrm{~m}^{2}$ sample fixture holds 36 Petri plates in which 144 Arabidopsis thaliana seedlings (4 per Petri plate) grew vertically along the surface of an agar gel. A probabilistic image analysis algorithm was used to locate the root of seedlings and a normalized gray scale variance measure was used to achieve focus by servoing along the optical axis. Rotation of the sample holder induced a gravitropic bending response in the roots, which are approximately $45 \mu \mathrm{m}$ wide and several millimeter in length. The custom hardware and
\end{abstract}

R. Subramanian $(\bowtie) \cdot$ N. J. Ferrier

Department of Mechanical Engineering, University

of Wisconsin-Madison, 2246 Mechanical Engineering Building,

1513 University Ave., Madison, WI 53705, USA

e-mail: ram@cae.wisc.edu

N. J. Ferrier

e-mail: ferrier@engr.wisc.edu

E. P. Spalding

Department of Botany, University of Wisconsin-Madison,

Madison, USA software described here accurately quantified the gravitropic responses of the seedlings in parallel at approximately $3 \mathrm{~min}$ intervals over an 8 -h period. Here we present an overview of our system and describe some of the necessary capabilities and challenges to automating plant phenotype studies.

Keywords High throughput · Visual servoing · Focusing · Plant phenotyping · Image segmentation

\section{Introduction}

A standard approach to determining the function of a gene is to discover the consequences of its mutation, which constitute the phenotype. For the reference species Arabidopsis thaliana, almost all of its approximately 25,000 genes have been mutated and seeds of most mutant plants are publicly available. However, phenotypes have been detected and described for perhaps as few as $10 \%$ of those genes. Even in those relatively few cases, better quantification of the phenotypes and discovery of additional phenotypes in the mutants would improve understanding of the affected genes. Adding the time domain to the analysis, allowing the development of a phenotype to be captured has proven very useful in determining details of gene function. Machine vision technology promises to be effective in the effort to improve plant phenotype research $[7,10,11,15,16,24]$, where the experiments call for micron level measurements of seedlings on time scales of minutes or daily measurements of large crop plants in a greenhouse.

To be effective at the genome scale, the measurement platform must have the capacity to characterize thousands of plants in weeks or months. This need for throughput cannot be at the expense of spatial or temporal resolution. Parallelizing data acquisition by replicating the imaging 


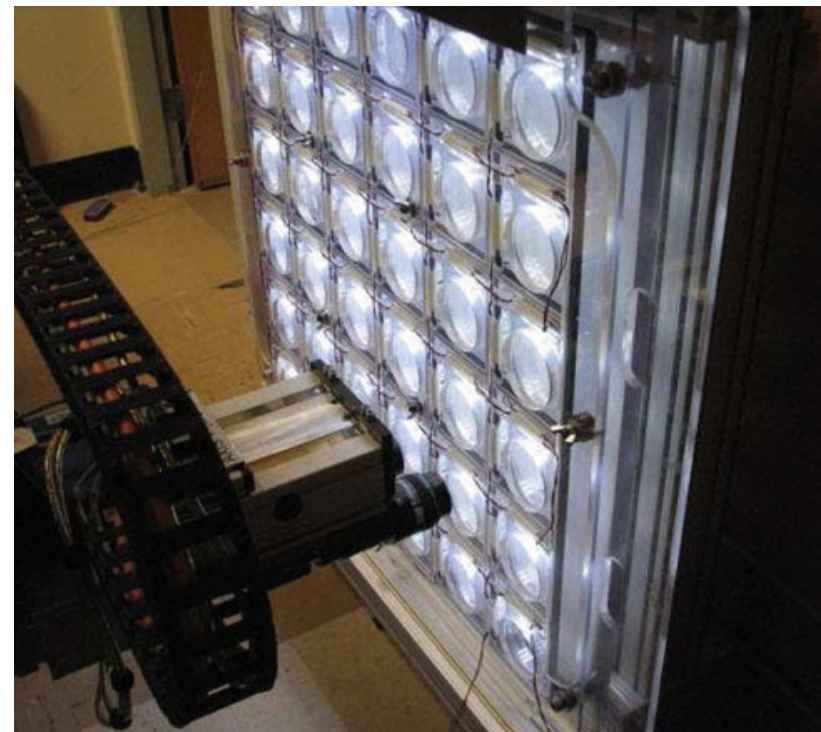

Fig. 1 Robot setup

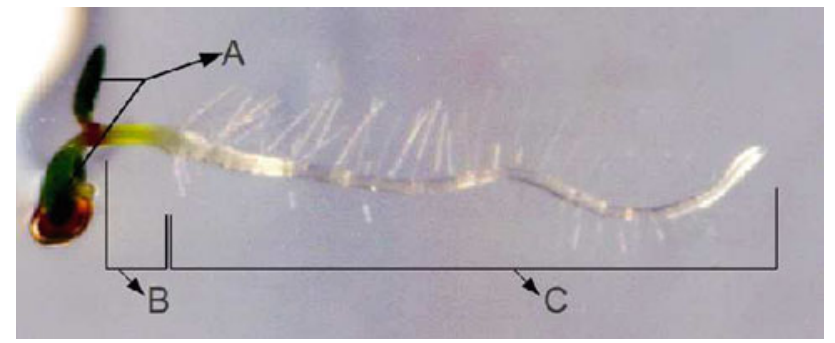

Fig. 2 An example of a seedling imaged under infra red light. Parts of the seedling are labeled: $A$ cotyledons, $B$ hypocotyl, $C$ root

system can increase throughput while keeping resolution and sample frequency constant, but the need to frame and focus each subject manually at the start of an experiment would be a daunting and highly repetitive task if carried out for a large number of imaging systems. This limits the scalability and increases the cost of this solution. As the images are acquired only at certain time points the imaging systems are idle for most of the duration of data gathering.

The repetitive tasks associated with collecting time series of consistently framed and focused images of multiple plants are well suited for automation by robotics. One robotic approach is to move the plants repeatedly through a fixedcamera imaging station [14]. However, moving the plants can potentially alter their growth and behavior at the fine scales intended to be captured and analyzed. This drawback can be avoided by moving the imaging device across a fixed grid of samples. For the moving camera solution to be effective, several machine vision application challenges must be solved. These include rapid and accurate visual servoing, object recognition, and real-time image-based focusing. This report describes the construction and performance of a gantry robot system (Fig. 1) that meets these challenges. The application presented is the tracking of root growth in seedlings of Arabidopsis thaliana (Fig. 2) after reorientation by $90^{\circ}$ to induce a curvature response known as gravitropism. The measurement throughput, spatio-temporal resolution, precision, and amortized cost of the robot system described here provides a strong rationale for adopting robotic platforms for plant phenotyping studies.

The remainder of this paper is organized as follows, Sect. 2 describes the hardware of the robotic system and the sample holding fixture. A brief description of a typical data gathering session is provided in Sect. 3. Section 4 provides details of the image processing used to locate the seedling root and root tip. Section 5 assesses the performance of the platform for monitoring a typical root gravitropic experiment. Section 6 provides an outline of future work.

\section{System description}

The function of the system described here is to locate numerous seedlings growing along the surface of a vertically oriented gel (agar) substrate using visual servoing and repeatedly imaging these seedlings in order to create a highly-resolved time series of images from which growth and behavior traits can be quantitatively extracted. The system consists of, a fixture that holds numerous seedlings vertically within illuminated Petri dishes and a visual servo controlled three degree of freedom gantry robot that locates each seedling and tracks its growth over a specified time.

\subsection{Petri dish containment unit (PDCU)}

The Petri dish containment unit holds 36 Petri dishes in a grid, laid out as a $6 \times 6$ pattern occupying a $1 \mathrm{~m} \times 1 \mathrm{~m}$ area, as shown in Fig. 3. This unit consists of a removable cassette, a fixture to hold the cassette in place and a removable backlight system. The Petri dishes are loaded onto the cassette vertically (six rows by six columns). Each dish contained two or four seedlings. For the purpose of this article we discuss the case with two seedlings per Petri dish (for a total of 72 seedlings per session), though success has been achieved with 4 and more. Every Petri dish is equipped with its own growth light and is isolated from the other Petri dishes in the grid to maintain uniform conditions (Fig. 4). When the whole cassette is secured onto the fixture it is illuminated with a back light. Ideally, the light used to obtain images of the seedlings would not affect their phenotypes. Light detection systems in plants are not appreciably influenced by wavelengths longer than approximately $750 \mathrm{~nm}$ so a backlight panel consisting of IR LEDs (output maximum at $850 \mathrm{~nm}$ ). The back light provides illumination during imaging. The whole Petri dish containment unit is designed to be modular. The lighting and 


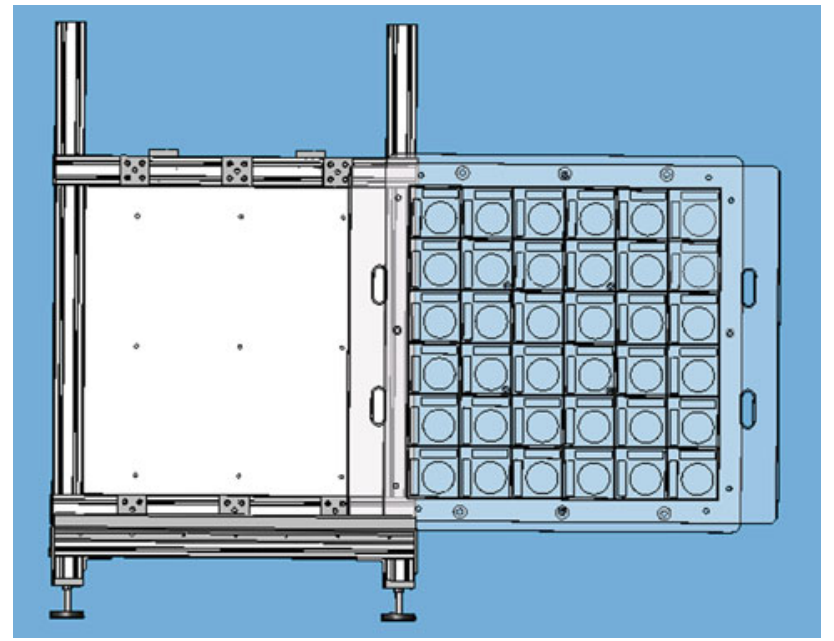

Fig. 3 Petri dish containment unit (PDCU)

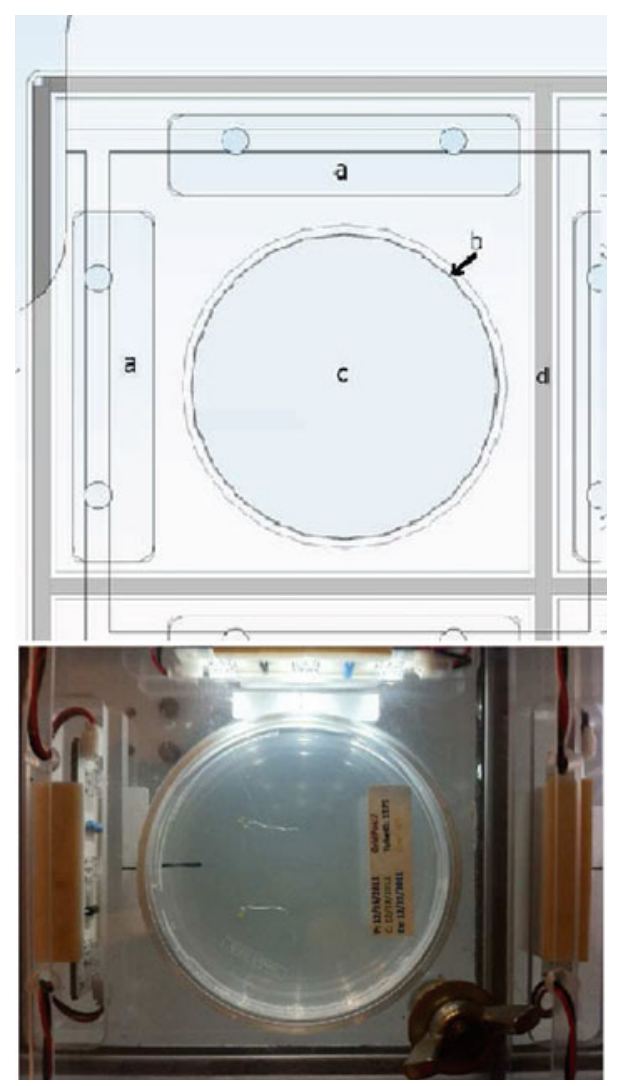

Fig. 4 Schematic and corresponding image of a single Petri dish in the PDCU. The components labeled are: $a$ LED grow lights, $b$ Petri dish, $c$ IR backlit region, $d$ Separators that isolate each Petri dish

the environment in each cell (around one Petri dish) can be changed for various experimental conditions. Each cell in the unit is equipped with two sets of lights, aligned $90^{\circ}$ from each other so that after rotating the holder, the seedlings remain illuminated from above.

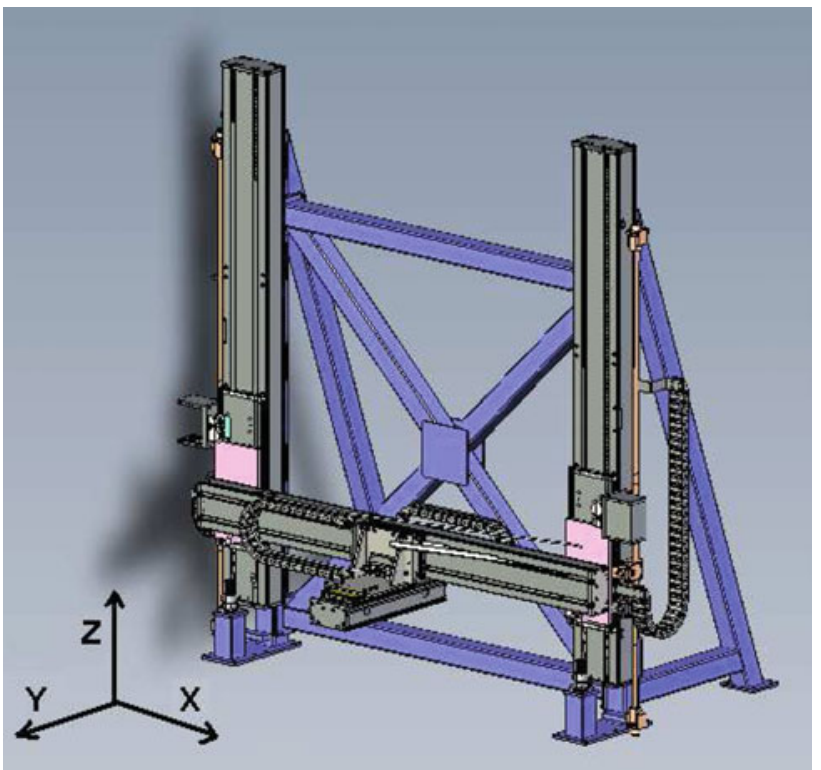

Fig. 5 Three axis gantry robot

Table 1 Gantry specifications

\begin{tabular}{lll}
\hline Degrees of freedom & & 3 \\
Workspace & $x$ & $1 \mathrm{~m}$ \\
Dimensions & $z$ & $1 \mathrm{~m}$ \\
& $y$ & $0.2 \mathrm{~m}$ \\
Accuracy & & $1 \mu \mathrm{m}$ \\
Repeatability & $0.1 \mu \mathrm{m}$ \\
Speed & $1 \mathrm{~m} / \mathrm{s}$ \\
Payload & 10 pounds \\
\hline
\end{tabular}

\subsection{Gantry robot}

The gantry robot has been constructed with 3-axis motion with a workspace large enough to servo two cameras along the vertical $(X-Z)$ plane to all positions of interest in the Petri dish cassette (Fig. 5). The dimensions, positional accuracy, and other specifications are listed in Table 1. Each axis is a direct drive linear servo motors with an individual position controller. One 4-axis master controller is used for global control of the gantry. The master controller uses a simple PID control, connected to the control computer via ethernet.

Two IR sensitive cameras are mounted on the end effector of the gantry. They are set up in a side-by-side configuration as shown in Fig. 6. Camera 1 with a low magnification lens $(14 \mu \mathrm{m}$ per pixel) is used for determining seed location and obtaining low magnification images (providing images of the entire seedling) and camera 2 with a high magnification lens ( $5 \mu \mathrm{m}$ per pixel) is used for servoing and image gathering (images of a $2-3 \mathrm{~mm}$ sections of the root). The cameras are calibrated using controlled motion of the gantry arm. The PDCU and the robot are positioned 


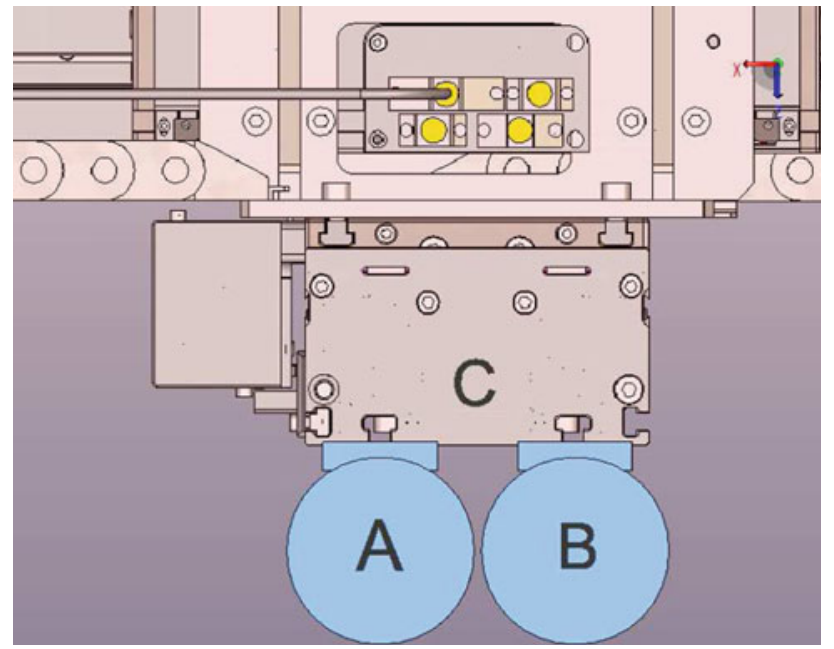

Fig. 6 A diagram of the two cameras installed on the end effector. $A$ Low magnification camera and lens. $B$ High magnification camera and lens. $C$ Robot end effector

facing each other (approximately parallel). If the PDCU and robot were perfectly parallel, calibration of the cameras using controlled motion of the gantry arm would allow precise location of Petri plate regions known to contain a seedling. However, the following issues prevent a straightforward hard-coded solution for object image acquisition. The optics required to obtain useful images of roots (e.g. Fig. 3) necessarily produce a depth of field as shallow as $1 \mathrm{~mm}$. Variation in the thickness of agar growth medium among the Petri plates is enough to cause many samples to be out of focus if a constant working distance was employed. The optics required to obtain the desired resolution necessarily produce a narrow field of view. A seedling may not be located within a predetermined 2 or $3 \mathrm{~mm}$ area (the typical width of a field of view) in each Petri dish because planting is a manual activity and sometimes seed germination and growth translates the seedling across the agar surface. Thus to ensure the sample is within the field of view, and in focus, we use visual servoing to track the root, center the tip in the field of view and ensure that is in focus.

\section{An example data gathering session using our robotic platform}

The seeds are grown in Petri dishes $(10 \mathrm{~cm}$ diameter). The Petri dishes are filled with agar (a commonly used growing medium) and (2 seeds per Petri dish) seeds are spaced far enough apart in a predetermined pattern, to minimize the interference of one seedling with another during growth
The dishes are kept at $4{ }^{\circ} \mathrm{C}$ temperature, for about 3-4 days, to promote and synchronize germination. The Petri dishes with germinated seedlings are stored vertically and illuminated from above during germination, promoting straight and vertical growth of the seedlings. Petri dishes are loaded on to the Petri dish cassette such that the seedlings will be imaged through the translucent agar. This prevents the condensation from drastically affecting the image quality.

The cassette is loaded onto the PDCU (described in Sect. 2) such that seedlings are now horizontal (all samples are rotated simultaneously as the cassette is loaded) to induce gravitropism. The task of gathering images of the root, as it reorients to the change in gravity stimulus, is broken into two stages. During the first stage (where the robot visits seedlings for the first time) visual servoing and focusing is performed to accurately localize the tip of the primary root of each seedling and ensure that it is in focus (while simultaneously saving the images). The location of each root tip is recorded for use in the second stage. The second stage of data collection involves collecting images of the root tip over a period of 5-8 h. If at any point the root grows out of the field of view, the robot will servo again to update the location of the root tip (for $5-8 \mathrm{~h}$ sessions most roots stay within the field of view). All the collected images are organized as time sequence stacks for offline analysis, The manual or stationary camera system would require similar preparation of the seeds/seedlings, but would require one seedling to be placed in each Petri dish. Each Petri would have to be placed in front of each camera and manually adjusted for focus and position to ensure that the root tip was within the field of view. Prior to the start of the data gathering session, the top cover of the Petri dish would require wiping to remove condensation from the lid. In the automated system, we image the seedling through the transparent growing medium to prevent the condensation from interfering with the imaging, thus the condensation does not pose a problem to our system.

Data collection is completely autonomous once the cassette has been loaded, resulting in one root per image per time point. The seedling images shown in Fig. 7 have been manually cropped to save space in this article. Fig. 7a shows a time lapse of a single seedling as it responds to gravistimulus.

Figure 8 consists of two plots overlaid on each other. They correspond to the values obtained from images of the seedling shown in Fig. 7a over a 5-h period. The color coded 2-D surface plot shows the curvature measured along a $1 \mathrm{~mm}$ region $100 \mu \mathrm{m}$ behind the root tip (along the ordinate) at each time point (along the abscissa). Typically the region next to the root tip is of interest (it is the region where the growth occurs and bending is generated), the plot only shows the region of interest. The second plot shows the angle (ordinate) of the root tip with respect to the horizontal at each of the same time points. These measurements were extracted using techniques from [15]. 


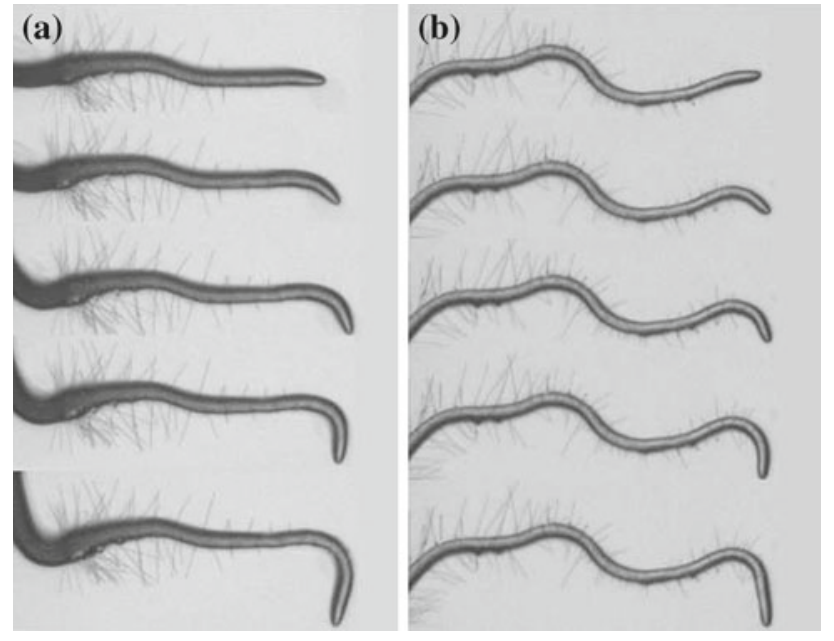

Fig. 7 Seedling responding to gravistimulus. Each image shows the same root at different time points over a period of $4.5 \mathrm{~h}$. These are the images obtained after servoing and focusing have been performed

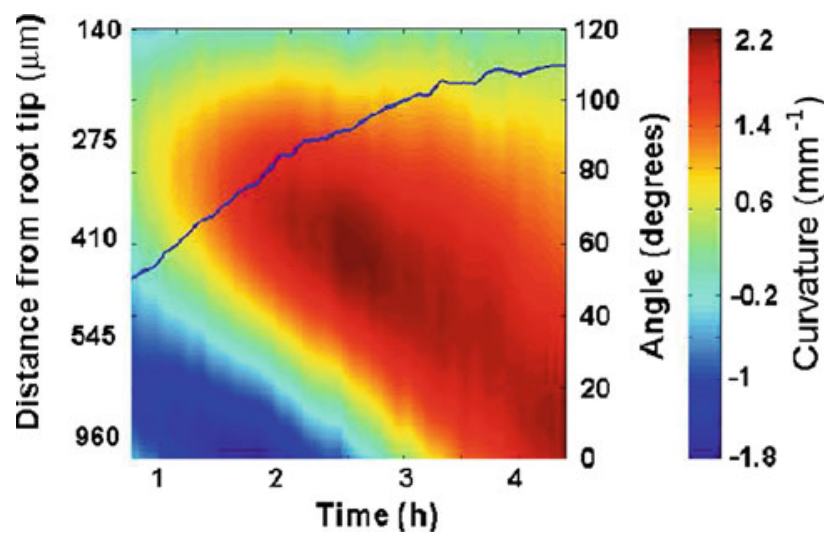

Fig. 8 The color coded plot shows the curvature of the region of the root close to the tip (within $1 \mathrm{~mm}$ ). The line plot shows the angle of the tip (with respect to the horizontal) over the same time period

\section{Image processing for visual servoing}

To increase the time course density of the gathered data the servoing is performed once at the start of the data gathering session to locate the seedlings. The servoing process is repeated only if the seedling's primary root tip grows out of the field of view. The servoing loop used is a simple image based visual servo scheme as described in [3]. In order to perform visual servoing, features need to be defined in the scene that can be tracked through the entire servoing process $[3,4,9]$. Typically, these features are image locations of markers/fiducials placed in the scene and are well defined and easy to extract from an image of the scene. Often dots with starkly different color or texture from the background/scene are used for markers. As the field of view and depth of field of our camera is as small as $1 \mathrm{~mm}$, respectively, markers would need to be engineered to be very small and very close to the seedlings. Placing them would be very tedious and the markers could interfere with the biology. Primarily, because there may arise a situation where the markers may interfere with the biological process being observed (blocking the root growth, etc). Even if successful, all the required markers may not be visible in the field of view. Recent work, [21,22] has shown that image moments can be used as features for servoing, Avoiding the need for markers placed in the scene. We define the servoing task as bringing the root tip close to the center of the field of view. This will involve the following steps:

- Locating the seedling.

- Identifying the root.

- Bringing the root within the depth of field of the lens.

- Determining the location of the root tip.

- Keeping the root within the depth of field of the lens.

For our system we have calibrated the cameras and the robot motion to the PDCU. Also, in a Petri dish the seeds are planted one to each quadrant (sometimes only top 2 of the 4 quadrants are used), thus gathering has been tested with 144 or 72 seedlings. Under ideal conditions, if the exact location of the seed was predetermined in the Petri dish, it would be enough to perform the above mentioned steps without visual servoing. But, owing to the small size of the seedling's root (approx $7 \mathrm{~mm}$ long and 40-45 $\mu \mathrm{m}$ wide) along with the small field of view and depth of field, small errors in calibration and/or alignment can cause the predetermined seedling locations to be inconsistent with the actual locations, a small motion (approx $2 \mathrm{~mm}$ ) in any direction can cause the root to be completely absent in the image. Hence a search will have to be performed to find and confirm that a seedling/root tip/root is present and is in focus. The robot moves the low magnification camera to the center of each quadrant of the petri dish to attempt a coarse localization of the seedling. A search is performed within the quadrant to find the seedling. Once the seedling and root is identified the robot visually servos along the root to the root tip with the high magnification camera.

The process of servoing is broken into two parts, rather than servoing in 3D [5,6]. Servoing is performed separately along the plane of the PDCU ( $X-Z$ directions) and along the direction normal to the PDCU ( $Y$ direction). Partitioning the motion in our system was straight forward as the robot is a 3-axis gantry with all motions being pure translations. Partitioning the servoing allowed us to break down our task into two tractable problems rather than performing them as one (which would lead to a situation where we may focus on an object not knowing if it were the root or tracking a region of an image although it might not be the root when brought into focus), but it still required identifying a root when it may 
be out of focus. Hence the critical tasks for servoing were to design:

(i) A technique to identify the root and its tip in the image, to provide a reliable target for the visual servoing algorithm even when the root and/or root tip is out of focus.

(ii) A technique to determine if the root/root tip is in focus and a scheme to arrive at the desired $Y$-axis location.

\subsection{Root and root tip segmentation}

The primary challenge that had to be addressed was reliably locating the seedlings in the Petri dish. In previous work related to image based phenotype analysis, segmentation operations and measurement extraction have made two fundamental assumptions:

(a) The feature of interest is always in the field of view.

(b) The feature of interest is in focus.

In the static setup described in [1], for example, the experiment involved determining the location of the root tip and manually focusing on it so the above assumptions were valid. In $[15,23]$ the authors reduce manual effort by bringing more seedlings within the field of view. This is done by either reducing the spatial resolution or bringing many seedlings very close together, introducing a new set of complications caused by seedlings crossing over one another. In our case, we needed to locate the seedling when it was not within the field of view and autonomously adjust the camera position and focus accordingly.

Our solution utilizes two cameras. One camera with low magnification capable of obtaining a large field of view (most of one quarter of the Petri dish). Although this makes the task of finding the seedling more tractable it introduces a set of problems. The quality of these images was often low enough to create challenges. Condensation on the lid of the Petri dish could cause dark regions that a threshold operation would convert into potential objects of interest, or reduce the contrast on which any segmentation process operates. Artifacts such as scratches on the agar surface or Petri dish could appear similar to roots. The illumination would not be uniform over the entire image. Figure 10 presents some typical images that the vision system encounters. Once the robot has completed the servoing task and performs a focusing step most seedling images resemble Fig. 9. Initial attempts were made to search for the seedling in and around the expected location in the vicinity of the seeds. This approach worked well to locate the seedling using only the high magnification camera. But it proved to be time consuming and significantly impacted the throughput of the system. Thus this technique was replaced by the two camera technique.

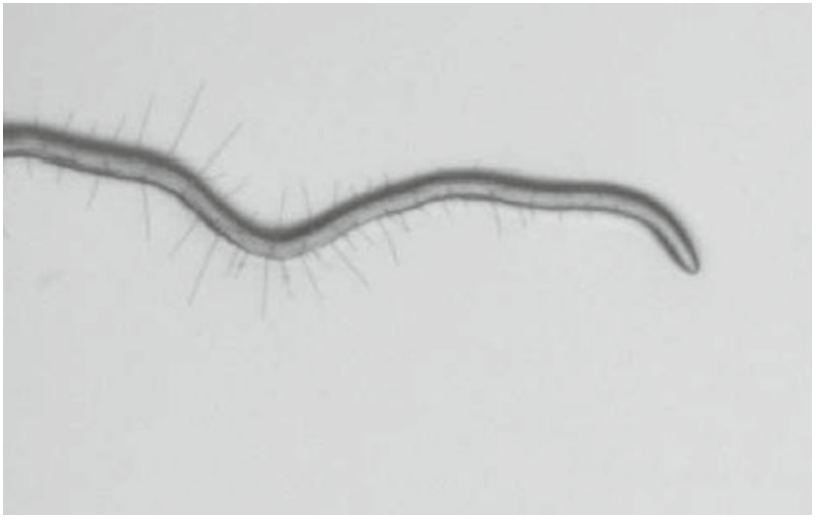

Fig. 9 An image of a seedling root responding to a change in gravity stimulus (after servoing and focusing)

The seeds are known to be in each quadrant of the Petri dish. The robot moves the low magnification camera in front of each quadrant of the Petri dish and the seedlings are initially identified in the low magnification image. The higher magnification camera is moved to the identify location and subsequently visually servo along the root to the root tip (the same image analysis routine was used for identification and segmentation for all images and both cameras).

The following section provides a detailed description of root segmentation procedure, which can identify the region of the image that is most likely to contain the the root even when it is not in focus (steps shown in Fig. 11). The segmentation task has been split into two operations. The first operation identifies regions of the image that can contain the root. The second operation uses heuristics and probabilities to identify the region with the highest amount of rootness. This segmentation procedure is used to identify the feature for the servoing task.

\subsubsection{Per pixel probability assignments}

Each pixel in the image is assigned a probability using a Bayesian framework. The probability assignments make two assumptions:

(a) The root is darker than the background.

(b) The root occupies at most $3 \%$ of the entire image.

The first assumption is because of the imaging modality. The root is opaque IR light. The root region contains darker gray levels than non-root regions. The second assumption is based on the magnification of the lens being used, the field of view available, and, the typical size of the Arabidopsis seedlings. The value $3 \%$ is obtained in cases where the root stretches across the entire image.

These two assumptions help define the likelihood and prior terms in the Bayes equation. We define these terms and 

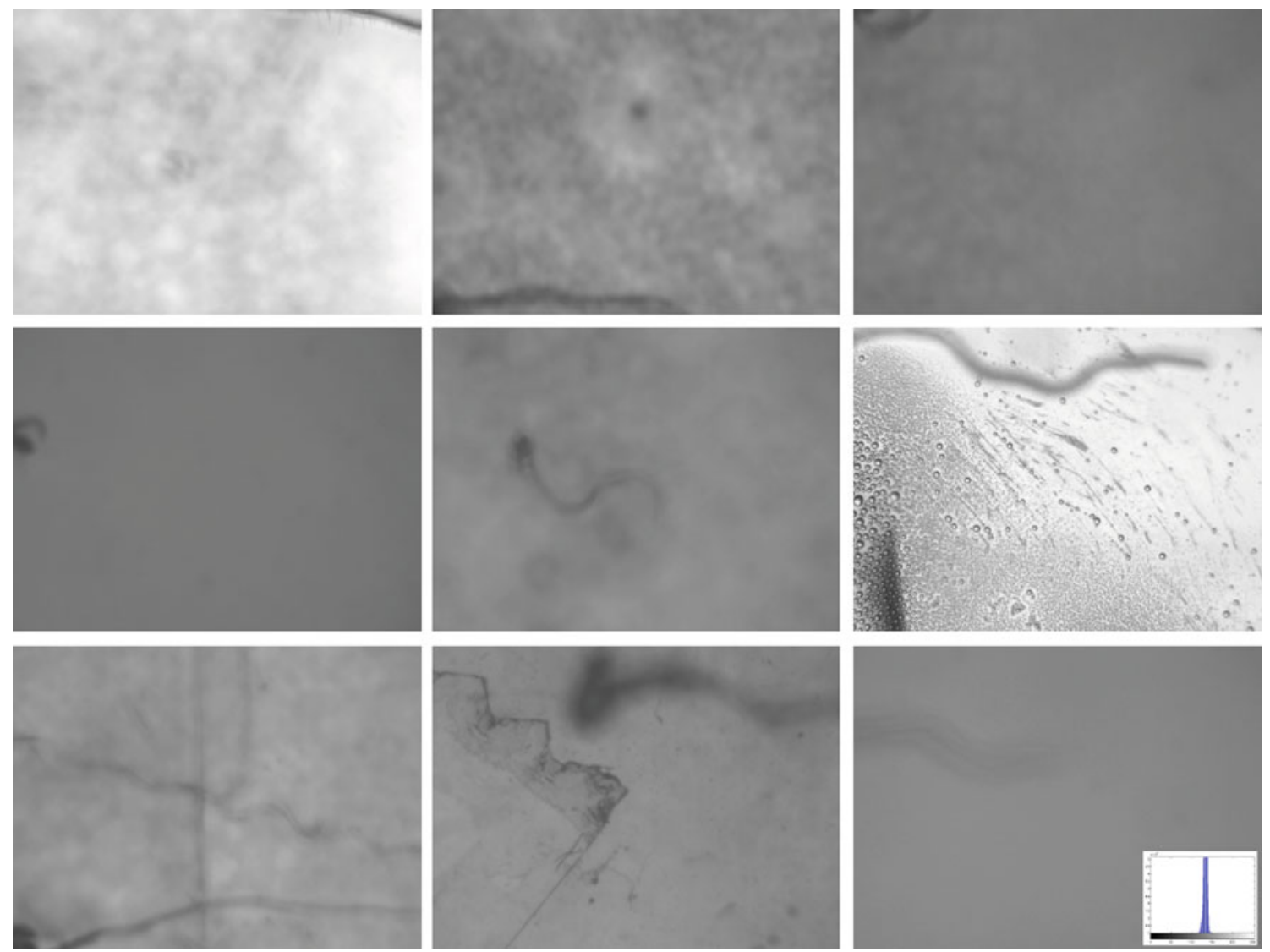

Fig. 10 These images depict some of the worst case scenarios observed when performing the task of visual servoing, (i.e. when the robot moves to a Petri dish location to find the seedling and root tip). The cause can be attributed to scratched on the Petri plate, condensation build up within

the probability assignments in formal terms below. We use a single channel image $I$, of size $m \times n$, with pixel intensities at pixel location $x, y$

$I(x, y)=g$

where

$x \in[0, m-1], \quad y \in[0, n-1], \quad$ and $g \in[0,255]$.

We can estimate the probability of the occurrence $g(p(g))$ as the expected value of $g$ (where $E$ is the expected) from the histogram of $I$.

$p(g)=E[g \mid I]$.

The probability of finding a root in an image based on its relative size in the image, $p(r)$ is 0.03 . This value was estimated empirically from a collection of images of the root acquired a priori. This value is required to help bias our probability estimate. The final probability assignments are not very sensitive to this value, Sect. 5 discusses the sensitivity of final segmentation to $p(r)$. the plate, or a scratch an non smooth surface on the growing medium. In contrast with the "ideal" image that we usually expect as shown in Fig. 9, these images represent the potential challenges in identifying the root

Finally we describe the likelihood function, that will estimate the probability of a pixel belonging to the root having a gray level $g$, based on our first assumption can be written as

$p(g \mid r)=\left\{\begin{array}{cl}1-\frac{g}{g_{\text {avg }}} & : g \leq g_{\text {avg }} \\ 0 & : g>g_{\text {avg }}\end{array}\right.$

where $g_{\text {avg }}=E[g]$, and if $g=0$ then $p(g \mid r)=0$. We discuss the reasons for our choice of linear model for $p(g \mid r)$ in Sect. 5.2.2. Using the terms defined above for the priors and the likelihood we can compute the conditional probability $p(r \mid g)$, probability of a pixel, $I(x, y)$, belonging to the root given it has a gray scale value $g$

$p(r \mid g)=\frac{p(g \mid r) p(r)}{p(g)}$.

Once the probabilities are assigned for every pixel we construct a probability matrix with each entry containing the probability of the pixel in the corresponding location in the image. This matrix is used in the next stage to identify the root (Fig. 11). 
(a)

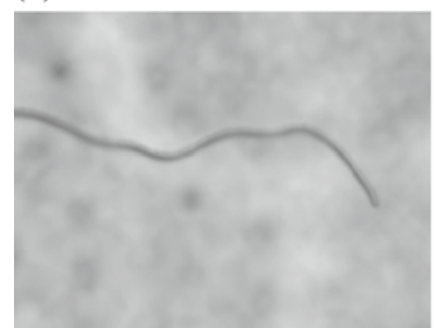

(b)

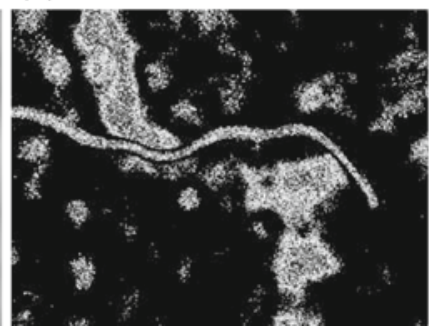

(c)

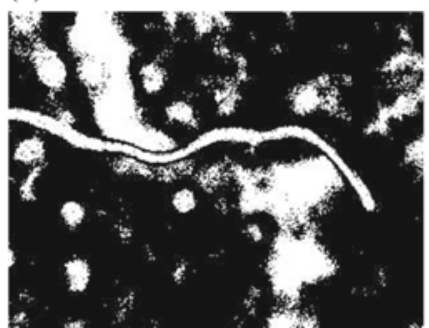

(d)

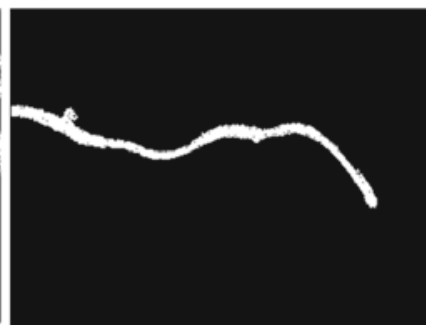

Fig. 11 The images show the output of the different steps in the segmentation process. a Original image, b pixel probability image, $\mathbf{c}$ binarized image and $\mathbf{d}$ segmented image

\subsubsection{Heuristic root selection}

From the previous step we obtain a probability matrix. From the original image we remove all pixels with $p(r \mid g)=0$. The remaining pixels cluster together forming patches in the image. In most cases, images with good contrast, the pixels form just one patch. This patch is considered to be the region which best represents the root. In cases with poor image quality many patches are formed. Poor image quality is a result of factors highlighted above. At this stage numerous techniques to exclude unwanted pixels can be used. From analysis performed we note that any adaptive threshold technique will work to isolate the high probability regions. A fixed thresholding technique works only in some ideal cases. For our purposes we choose to remove pixels that have $p(g) \cdot p(r \mid g)>p(r)$. This follows from the fact that background pixels will be present in large numbers. This threshold helps in removing low probability pixels from gray levels that occur in large numbers in the image. The removal of these pixels produces isolated regions in the image from which the region containing the root needs to be identified. Section 5 provides a brief discussion of algorithm's sensitivity to the value of $p(r)$.

Each patch is assigned a probability equal to the average of all its pixel probabilities. Heuristic driven weight assignments are made for each patch. The heuristics use information from basic moment analysis (area, major, minor axis) on the patches. The larger, longer and thinner patches that are more horizontal are assigned higher weights. We term this weighted probability the rootness of the patch. Finally, the patch with the highest rootness, weighted probability is chosen as the most likely root patch. If there are $n$ patches in the image we can formally define the rootness as

$w_{1}^{i}=1-\frac{\operatorname{minor}_{\operatorname{axis}^{i}}}{{\text { major } \operatorname{axis}^{i}}^{i}}$

$w_{2}^{i}=1-\frac{\text { Orientation }^{i}}{90}$

$w_{2}$ is set up to look for a horizontal root as the roots will be horizontal when the cassette is rotated and placed in the PDCU. Also the root identification needs to be carried out during the first pass. All angles are computed as the values in the first and fourth quadrant and thus the orientation is always between $0^{\circ}$ and $90^{\circ}$.

$w_{3}^{i}=\frac{\text { area }^{i}}{\sum_{i=0}^{n} \operatorname{area}^{i}} \quad$ where $i=1 \ldots n$

For every patch $i$, we average the probability over all $m$ pixels

$$
\begin{aligned}
& p_{\text {avg }}^{i}=\frac{\sum_{j=0}^{m} p\left(r \mid g_{j}\right)^{i}}{m} \quad \text { where } j=1 \ldots m \\
& p_{\text {root }}^{i}=w_{1}^{i} \cdot w_{2}^{i} \cdot w_{3}^{i} \cdot p_{\text {avg }}^{i} \\
& \text { rootness }=\max \left(p_{\text {root }}^{i}\right) .
\end{aligned}
$$

The patch designated as having the maximum rootness is labeled as the root (the most likely root patch) used for the servoing algorithm. The robot servos to the tip of the patch (bringing it to the center of the field of view). If the root does extend beyond the field of view, the robot servos until a tip is reached and positions the near the center of the field of view.

\subsection{Focusing}

Focusing needs to be performed to ensure that the root tip images, are as clear and crisp with minimal amount of blur as possible. Ideally, if no alignment errors exist between the gantry and the PDCU and the the PDCU is perfectly flat without any flexure and the agar growing medium in each Petri dish around the seedling is the same thickness, focusing would not be necessary. But, if the PDCU is misaligned by more than $0.2^{\circ}$ more than half of the seedlings will be out of the depth of field of the lens. Thus a system for auto focusing is required. Auto focusing techniques fall broadly into two categories, active and passive. Active focusing techniques rely on additional sensors like, lasers, SONAR, etc., to provide additional range information. While, passive focusing techniques employ software based techniques to obtain the same range information directly from the image. Typically, multiple images are obtained and a quantitative measure (focus measure) of the extent of focus (or defocus) is 
computed from the image. In both cases a search will need to be performed to determine the location of the best focused image. In our case, we chose to use a passive focusing technique, to avoid using any additional sensors or equipment being added to the gantry.

In the description of the segmentation algorithm (Sect. 4.1) we have shown that the servoing can be performed with images of the root that may be out of focus. This section describes the technique used for searching (along the $Y$-axis of the gantry) for a focused image of the root tip. Unlike the search of the root tip (described in Sect. 4.1), where the search is performed in image space (along the $X-Z$ plane of the gantry), here the search is performed in the space of the focus measure. Instead of using the entire image, the image patch with the highest rootness measure is used. The process involves determining the value of the focus measures at a few different positions (along the $Y$-axis) and estimating the position that would provide a maximum focus score. The description of the focus measure and the search technique used are presented below.

\subsubsection{Focus measure}

From the work in $[8,13,17,20,25]$, it is shown that most focus measures try to measure image properties like gray level image gradients, image contrast or frequency content. In essence they are a measure of the extent of the blur in the image. From a review of previous work on image based focusing, the most commonly used focus measures were identified and are listed below. If we have an image $i$ with height $H$ and width $W$ with $i(x, y)$ representing the gray scale value of a pixel in $i$ located at row $y$ and column $x, \mu$ is defined as the mean gray level of the image $i$, we can define the following focus measures as:

a. Tenenbaum Measure (Tenengrad) - the image is convolved with a Sobel operator and the sum of the square of the gradients in the $\mathrm{x}$ and $\mathrm{y}$ directions is computed.

$$
F_{\text {tenengrad }}=\sum_{H} \sum_{W} S_{x}(x, y)^{2}+S_{y}(x, y)^{2}
$$

where $S_{x}(x, y)$ and $S_{y}(x, y)$ are the results of convolving a window around $i(x, y)$ with the Sobel operators in the $x$ and $y$ direction.

b. Variance-computes the variations in gray levels in the image.

$$
F_{\text {variance }}=\frac{1}{H \cdot W} \sum_{\text {height }} \sum_{\text {width }}(i(x, y)-\mu)^{2}
$$

c. Normalized variance-similar to the variance measure except the value is normalized with $\mu$.

$$
F_{\text {normvariance }}=\frac{1}{H \cdot W \cdot \mu} \sum_{\text {height width }} \sum_{(i(x, y)-\mu)^{2}}
$$

d. FFT Magnitude-FFT based methods use the log magnitude values of the image in frequency space. The strength of the low frequencies and high frequencies are computed.

$$
F_{\mathrm{FFT}}=\frac{1}{h \cdot w} \sum^{h} \sum^{w} \log (|\Re e \mathbb{I}(x, y)|)
$$

where $\mathbb{I}=\operatorname{FFT}[i] . h$ and $w$ determine the region in frequency space (cut off frequencies) within which the frequency strengths are averaged.

Focus measures mentioned above, when evaluated on images of roots, performed in a similar fashion. Figure 12 shows sample plots of the focus measures, it can be seen that the focus measures produce higher values as $\mathrm{Y}$ position that is closer to the best focus position (indicated by the vertical line). The $F_{\mathrm{FFT}}$ measure was found to be very consistent but always slightly shifted than the others. On further analysis it was found that this was due to the presence of root hairs which are very small compared to the root and thus contribute heavily to the higher frequency response. But this measure is computationally intensive, thus negatively affecting throughput. Owing to ease of computation and simplicity the normalized variance measure was adopted. This focus measure was subsequently used to search for the gantry position with the best focus.

\subsubsection{Searching technique for focusing}

Using the focus measures chosen in the previous section a search needs to be performed to determine the location at which the best focused image of the root can be obtained. The techniques used to search for the best focused position work under the assumption that the scene contains only one object of interest and, within the search range there exists a single position where the object is in focus (i.e. the focus measure will produce one maxima within the search range). Thus, in theory, the focus measure values can be modeled as a quadratic function (as used in $[8,13]$ ) and hence the maxima can be determined by obtaining the focus measure at three different gantry positions and the location of the maxima can be directly estimated (shown in Fig. 14). The estimated location of the maxima is indicated by $\max _{q}$. This technique of using a quadratic function as a model and determining the maxima has been the most common approach, 


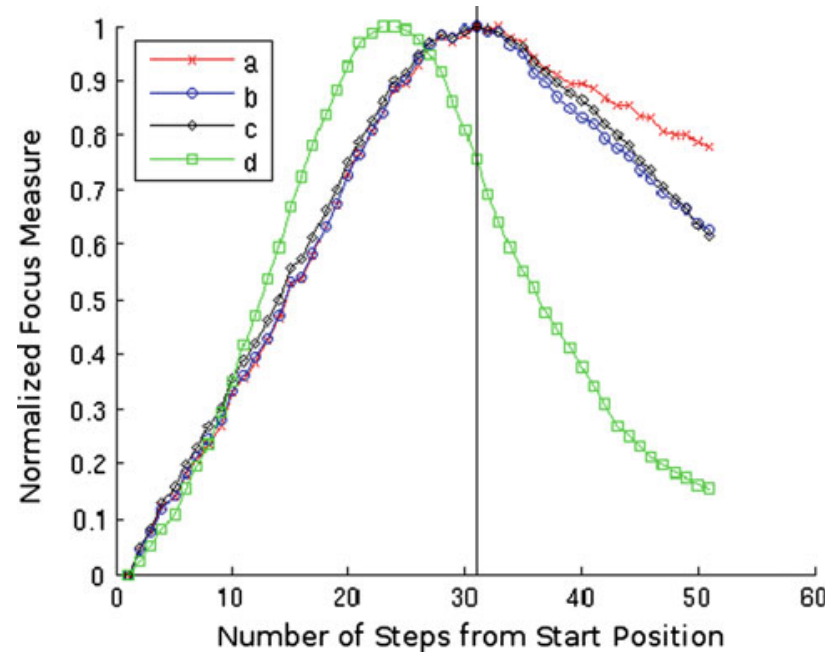

Fig. 12 Plot showing the four focus measures. $a$ Tenengrad, $b$ normalized variance, $c$ variance and $d$ FFT measure. Each measure has been individually scaled to range between 0 and 1 . The abscissa shows the number of steps taken from a starting depth at step 0 . The ordinate shows the scaled values of the frequency measure

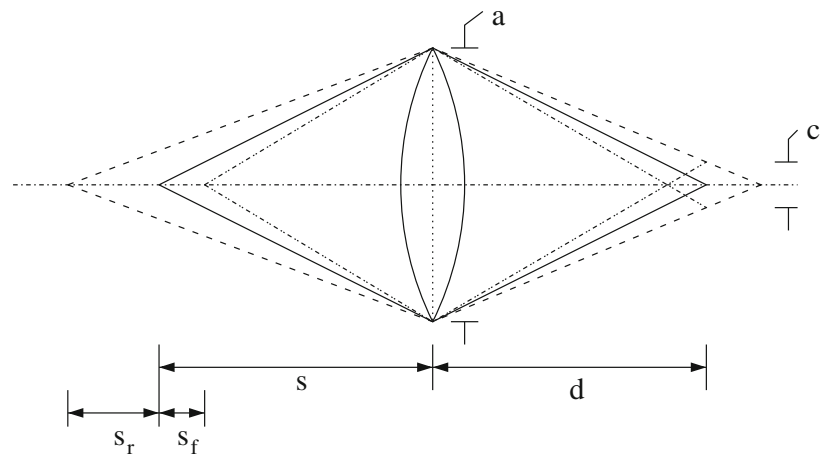

Fig. 13 Thin lens system with focal length $(f)$, aperture (a), object to lens distance $(s)$, image to lens distance $(d)$, depth of field in front and rear of the object $\left(s_{f}\right.$ and $\left.s_{r}\right)$

$[8,12,13,17,19,20,25]$. From optics literature, given the properties of the lens we can compute the depth of field for varying lens to object distances, using the relation in Eq. (7) obtained from Fig. 13.

$\frac{1}{s}+\frac{1}{d}=\frac{1}{f}$

where $f$ is the focal length, $a$ is the diameter of the lens, $s$ is the distance of the object from the lens and $d$ is the distance away from the lens at which the image is formed. $c$ is the circle of confusion, it is a value based on the perception of the human eye. The typical value use by photographers is $0.033 \mathrm{~mm}$. The value indicates the maximum size of a blurred image of a point object at which the human eye no longer perceives the image as a point (i.e. perceives it as blurred). The values $s_{f}$ and $s_{r}$ are distances in front of the object and behind the object up to which the circle of confusion is smaller than $c$.
Using Eq. (7) and the Fig. 13 we can write the following relations.

$$
\begin{aligned}
& \frac{1}{s+s_{f}}+\frac{1}{d\left[1+\frac{c}{a-c}\right]}=\frac{1}{f} \\
& \frac{1}{s+s_{r}}+\frac{1}{d\left[1-\frac{c}{a+c}\right]}=\frac{1}{f}
\end{aligned}
$$

on re-arranging, we have

$$
s_{f}=\frac{s c(s-f)}{f a+c(s-f)}, \quad s_{r}=\frac{s c(s-f)}{f a-c(s-f)}
$$

From Eq. (10) it can be seen that the value of $s_{r}$ will be greater than $s_{f}$. This produces an asymmetry, the depth of field in front of the object will be always less than the depth of field behind the object. Thus the values obtained from a focus measure cannot be modeled accurately as a simple quadratic curve.

In the current application, the peak of the focus measure may not occurs at the center of the search space. In most situations one set of sampled (either the first two or the last two) points tend to be closer to the peak than the other. To indicate the problems that arise, we choose a dataset where we have acquired images through a full range of focus. For the purpose of our discussion we simulate a few different cases that will be encountered, by varying the choice of sampled points and showing to the reader the variations that occur to the fit and the estimate of the location peak focus.

When comparing Figs. 14 and 15 it can be noted that the curve $q$ changes drastically when the choice of sampled locations change from $p_{2}, p_{3}, p_{4}$, in Fig. 14 to $p_{1}, p_{2}, p_{3}$, in Fig. 15. Making the quadratic estimation of the focus measure maxima unstable and sensitive to noise (in the computed focus measure). In addition quadratic model is not an accurate representation of the occurring phenomenon.

From previous work $[13,17,19]$, the two most preferred techniques, are (a) Global Search, (b) Fibonacci Search. The global search technique searches the entire depth range with fixed step size to determine the location of the maxima in the focus measure. This is very time consuming and the least preferred option in our case as it will affect throughput significantly. The Fibonacci search has been shown to be optimal, as mentioned in [13], but even this scheme requires at least 10-15 images to be captured to determine a stable maximum. The search speed can be increased by using larger step sizes, at the expense of accuracy. In some cases, as discussed in [17,19], the search is started with a large step size and repeated in a chosen region with smaller step sizes. All of these techniques adversely affect the throughput of our entire system. This is because, if more images are need to determine the focus, more time is spent focusing thus fewer seedlings can be observed in a fixed amount of time. 


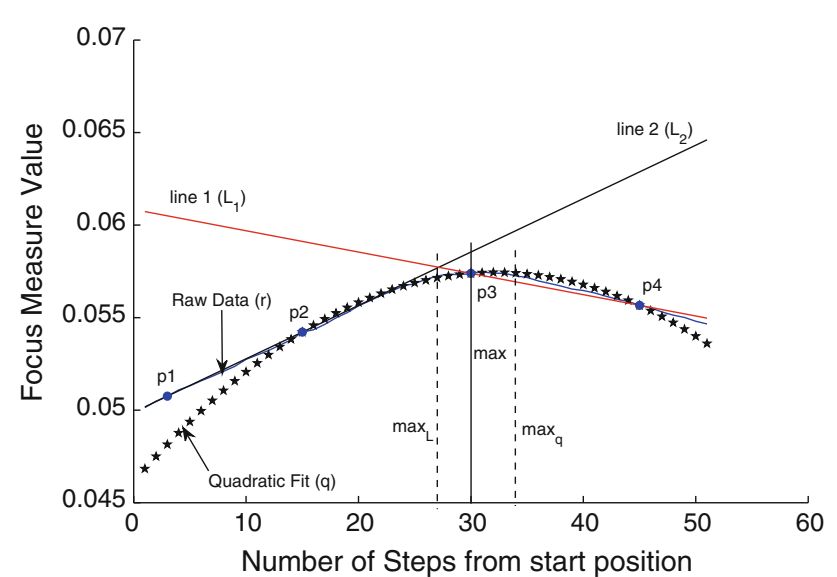

Fig. 14 Plots comparing two search techniques. The raw data $(r)$, the quadratic fit $(q)$ and the linear fit $\left(L_{1}\right.$ and $\left.L_{2}\right)$, when using locations $p_{2}, p_{3}$ and $p_{4}$ are shown. The estimated location of the focus measure maxima is at $\max _{L}, \max _{q}$ and $\max$, for the linear fit, quadratic fit, and manual focusing. The depth of field is about 5 steps and step size is about $50 \mu \mathrm{m}$

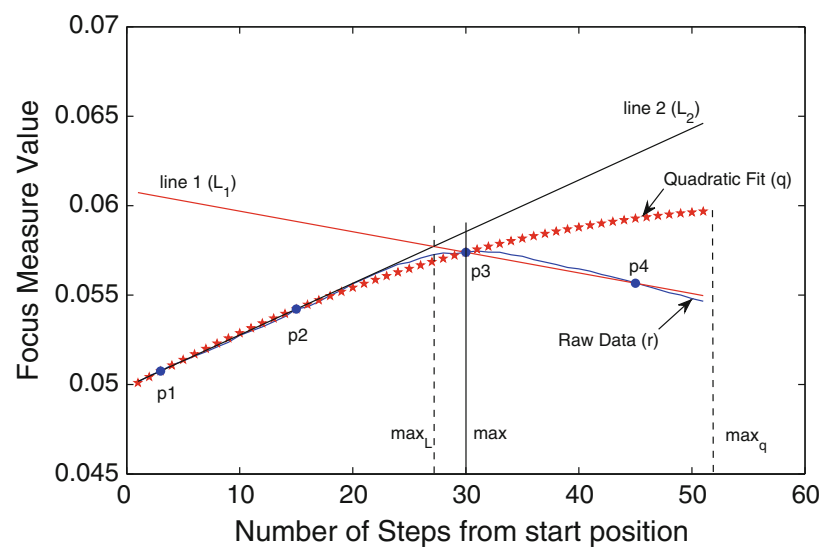

Fig. 15 Second plot comparing two search techniques with a different set of sample points. The raw data $(r)$, the quadratic fit $(q)$ and the linear fit $\left(L_{1}\right.$ and $\left.L_{2}\right)$, when using locations $p_{1}, p_{2}$, and $p_{3}$ are shown. The estimated location of the focus measure maxima is at $\max _{L}, \max _{q}$ and $\max$, for the linear fit, quadratic fit, and manual focusing. The depth of field is about 5 steps and step size is about $50 \mu \mathrm{m}$

Based on the discussion in the preceding paragraph we chose to model the focus measure function as two lines whose slopes correspond to the increasing and decreasing trends in the focus measure. This model will better represent the asymmetric nature of the depth of field. We also show (in Figs. 14, 15, 16) that this model is more resistant to noise in the focus measure and is less sensitive to the sampled positions. The intersection point of the two lines will correspond to the peak value, from which the required position can be estimated. To construct the model, the focus measure needs to be obtained from four images obtained at four different locations. The gantry can be moved to these locations and the focus measure is computed from the obtained images. The first two images and the last two images are captured

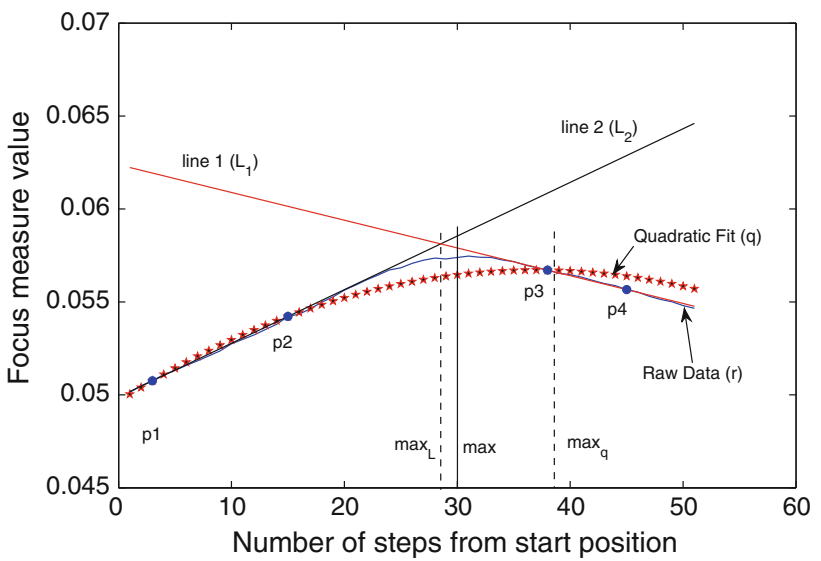

Fig. 16 Plots comparing the quadratic fit and linear fit. Shows the raw data, the estimate when using a quadratic fit and the line fit. When the sampled locations are changed the linear fit estimate of the peak location is more stable than the quadratic fit estimate

with the small change in the $\mathrm{Y}$ position of the gantry. The $\mathrm{Y}$ position change between the second and third image is comparatively large. Two straight lines are fit, one to the first two points and another to last two points. The intersection of the two straight lines are computed. This intersection point will estimate the location the gantry needs to move to to obtain a focused image of the root. This process is shown in Figs. 14 and 15. The lines $L_{1}$ and $L_{2}$ are fit using the focus measure values obtained from locations $p_{1}, p_{2}, p_{3}, p_{4}$. When comparing Figs. 15 and 16, it can be seen that unlike the quadratic fit estimate the line fit estimate is more stable when the chosen sample locations are changed ( $p_{3}$ is shifted) and is less sensitive to noise in the focus measure values. This technique has been successfully used to acquire data from more than 4000 seedlings so far.

This method will not guarantee optimal position estimation, as seen by the discrepancy between $\max _{L}$ and max, but will bring the gantry to a location such that the root is located within two depth of field of the lens. The advantage over other search techniques is that it requires far fewer images, hence less time to focus.

Using the above described servoing and focusing technique we have been able to successfully gather image data concurrently for up to 144 seedlings in one session.

\section{System performance evaluation}

In this section the performance (throughput) of the system is analyzed from two different view points, (i) The user (plant biologists) and (ii) The system design. In order to discuss each of these two points in detail we start by briefly describing the process of data gathering, which is broken into two stages. Stage 1, is the determination of the locations of the root tips 
for all the seedlings. The gantry visually servos to the tip of the root and records the locations. Stage 2 involves repeated visits to the recorded locations to capture the changes occurring at the root tip. Stage 1 is performed once per data gathering session and is time consuming, currently requires about 20 min to complete Stage 2 consists of multiple passes over the cassette to the recorded locations, with each pass taking $3.5 \mathrm{~min}$. The following equations provide a detailed break down of the time, for which the data gathering is performed. This will help discuss and better understand the performance and bottlenecks of the system.

$$
\begin{aligned}
& t_{\text {gath }}=t_{\text {pos }}+t_{\text {settle }}+t_{\text {cap }} \\
& t_{\text {gatherall }}=N * t_{\text {gath }} \\
& t_{\text {loc }}=t_{\text {focus }}+t_{\text {find }}+t_{\text {servo }} \\
& t_{\text {fp }}=t_{\text {loc }} * N \\
& t_{\text {tot }}=t_{\text {fp }}+n * t_{\text {gatherall }}
\end{aligned}
$$

\begin{tabular}{|c|c|}
\hline$N$ & Number of seedlings \\
\hline$n$ & Number of time steps to be captured \\
\hline$t_{\mathrm{pos}}$ & Time to move robot to a given position \\
\hline$t_{\text {find }}$ & Time to segment the root \\
\hline$t_{\text {settle }}$ & $\begin{array}{l}\text { Settling time provided to prevent motion } \\
\text { vibrations from causing image blur }\end{array}$ \\
\hline$t_{\text {servo }}$ & $\begin{array}{l}\text { Time to servo to region of interest } \\
\text { (root tip) }\end{array}$ \\
\hline$t_{\text {focus }}$ & Time to determine proper focus \\
\hline$t_{\text {cap }}$ & $\begin{array}{l}\text { Time to capture and store image data of } \\
\text { region of interest }\end{array}$ \\
\hline$t_{\mathrm{tot}}$ & Total time for data acquisition \\
\hline$t_{\mathrm{loc}}$ & Time to locate seedling \\
\hline$t_{\mathrm{fp}}$ & $\begin{array}{l}\text { Time to locate all seedlings on } \\
\text { the first pass }\end{array}$ \\
\hline$t_{\text {gath }}$ & $\begin{array}{l}\text { Time to gather one image from one } \\
\text { location }\end{array}$ \\
\hline$t_{\text {gatherall }}$ & $\begin{array}{l}\text { Time to gather one image from all } \\
\text { location in the cassette }\end{array}$ \\
\hline$t_{\text {onset }}$ & $\begin{array}{l}\text { Estimate of the time lapsed between the } \\
\text { change in stimulus and the seedlings } \\
\text { first response }\end{array}$ \\
\hline$t_{\text {complete }}$ & $\begin{array}{l}\text { Estimate of the time lapsed between the } \\
\text { change in stimulus and the seedling } \\
\text { reaches a steady state }\end{array}$ \\
\hline
\end{tabular}

A few points to note on the variables mentioned above. $t_{\text {pos }}$ depends on size and speed of the system and is fixed by design. The gantry robot with its high speed, large size and high accuracy, allows all motions to be less than 1 second, thus keeping $t_{\text {settle }}$ to be about 1 second. Considering the speed of camera and computer, $t_{\text {cap }}$ and $t_{\text {find }}$ each are much less than $1 \mathrm{~s}$, thus are not very critical for the analysis of the system performance.
5.1 Performance analysis: user perspective

From the user perspective (plant biologists), who define the biology to be observed and the length of the data gathering session, there are two primary concerns:

(a) In terms of timing - amount of time required to locate and image all the seedlings in the Petri dish cassette.

(b) In terms of yield - number of useful seedling data gathered per session (as described in Sect. 3).

The system is designed such that during the first pass over the cassette, time is spent locating and focusing on each seedling (Eq. 14) (in addition to the time spent collecting the image data, Eq. 12), as described above. This makes the first pass through the cassette longer than each of the other passes. For the subsequent passes the system requires a shorter, albeit finite, period of time. These two parameters $t_{\mathrm{fp}}$ and $t_{\text {gatherall }}$ implicitly effect the system performance for the user.

Equation 14 computes the amount time taken for the first pass, this specifies the lower limit of the biological process that can be observed. From Eq. 12, we can determine the total amount of time required gathering data for one full pass over the cassette. This will determine upper limit of the speed of the biological process. Any biological process that has an onset time $\left(t_{\text {onset }}\right)$ greater than $t_{\mathrm{fp}}$, but a completion time ( $\left.t_{\text {complete }}\right)$ smaller than $t_{\text {gatherall }}$ will be under sampled. Thus $N$, will have to be scaled based on the speed of the biological process. Currently, even with 72 seedlings planted in the cassette, $t_{\text {gatherall }}$ is approximately $3.5 \mathrm{~min}$ (the time gap between two successive images of a given seedling). This time resolution is much faster than a typical biological process, which are in terms of hours. Hence is not of critical concern to the throughput. This can be seen from the following equation.

For determining the number of seedlings, $N$, that can be observed in a session, we have the following constraints from the above discussion

$\begin{array}{ll}t_{\text {fp }}<t_{\text {onset }} & t_{\text {gatherall }}<t_{\text {complete }} \\ N<\frac{t_{\text {onset }}}{t_{\text {loc }}} & N<\frac{t_{\text {complete }}}{t_{\text {gath }}}\end{array}$

Using Eqs. 14 and 12 and rearranging we have Eqs. 17. As mentioned above, $t_{\text {complete }} \gg t_{\text {gath }}$, thus the value of $N$ is determined by Eq. 18, but for a generic gathering session we would pick the lower $N$ value.

$N<\frac{t_{\text {onset }}}{t_{\text {loc }}}$

Having derived an equation for the upper limit on the number of seedlings that can be imaged during a given session, the user will be interested in overall yield in the data gathered. This is described as the percentage of seedlings on the cassette that provided image data from which measurements are 
Table 2 Robotic system performance

\begin{tabular}{lrrr}
\hline Test \# & 1 & 2 & 3 \\
\hline Seedlings planted & 144 & 144 & 72 \\
Bad data/servo or focus error & 18 & 26 & 5 \\
Bad data/scratch on agar, & & & \\
$\quad$ improper germination, growth & 9 & 11 & 6 \\
$\quad$ outside depth of field & & & \\
Good data potentially available & 135 & 133 & 66 \\
Effective yield (\%) & 86 & 80.5 & 92.4 \\
\hline
\end{tabular}

reliably extracted and used. The effective yield takes into consideration that some seeds in the cassette may not have germinated or other external non system related issues. The effective yields for three sample gathering sessions are reported in Table 2.

\subsection{Performance analysis: system design perspective}

The previous section provided an analysis of the system from the user point of view and identified the operating limitation with respect to throughput of data gathered. In this section three other aspects of the system are discussed in detail namely effective yield, root segmentation and focusing. These effectively determine how well the system is working and have a significant effect on the throughput of the entire system.

\subsubsection{Effective yield}

The effective yield of a data gathering session is measured in terms of the useful data obtained from a data collection session, i.e. the cost of failure to collect useful data from a seedling in the PDCU. This is simply the performance adjusted, taking into account that some seedling locations had seedling deemed not "useful" or not "good". Data from a seedling is deemed "useful" or "good" if the root \& root tip can be identified and measurements can be reliably extracted. Thus providing information about the number of "good" seedlings for which data was not acquired. Table 2 lists three examples of a typical data gathering session and identifies the number of seedlings that were identified as "good" and number of seedlings the system failed to acquire. The seedling data that were not acquired fall into two categories, (a) Data unavailable due to external factors (not related to the robotic system) and (b) Data unavailable due to systemic failures (related to limitation of the hardware or software). These are itemized below.

Data losses due to external factors Some of the situations where failure to collect data is due to factors not related to the

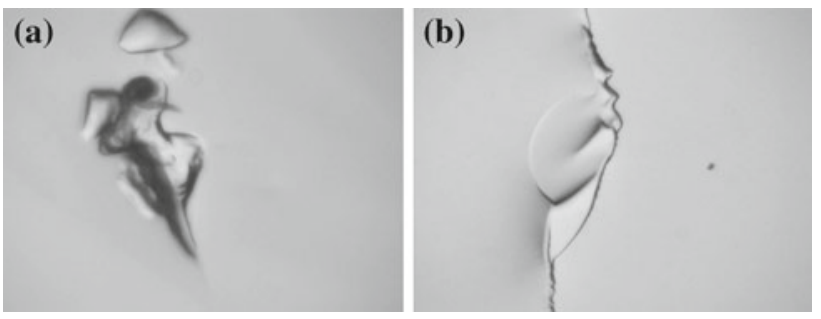

Fig. 17 a Scratches. b Peeling of agar

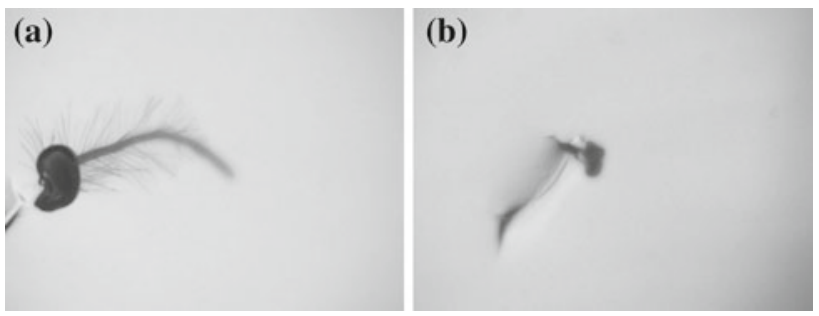

Fig. 18 a Growth out of focus plane. b Non-germinating seed

robotic platform are itemized below with examples shown in the figures. These are typically due to anomalies in the medium, scratches on the Petri dishes, seeds failing to germinate or seedling not adhering to the growing medium, etc.

(a) Scratch on agar medium-a scratch in the agar imaged in infrared light contains many features similar to a young seedling (about 2 days old). These are sometimes erroneously marked as seedlings (see Fig. 17a).

(b) Peeling of the agar medium-in some cases the agar peels away from the plate and thus produces a long root-like artifact, which the servoing system erroneously identifies as a root (see Fig. 17b).

(c) Root growth out of the depth of field-this phenomenon occurs in two forms, both of which cause focusing problems. The first, the root during the first day or two of the germination process has grown into the agar medium rendering one part of the root at one depth and the other at another (Fig. 18a). The second case, occurs when the root grows into the agar during data collection. Both cause the data gathered to be out of focus, in some cases almost impossible to use.

(d) Non or late germination-some seedlings do not germinate or germinate much later than the majority of the other seedlings (see Fig. 18b). This can result in no significant root-like feature being present and can cause the segmentation routine to identify a scratch as a the most likely root region.

(e) Thin roots-these are seen in a few cases and tend to be half or quarter the thickness of healthy regular roots and are generally rejected as bad data (Fig. 19).

(f) Random growth of roots - there are cases where the root or the hypocotyl curl around, which makes any 


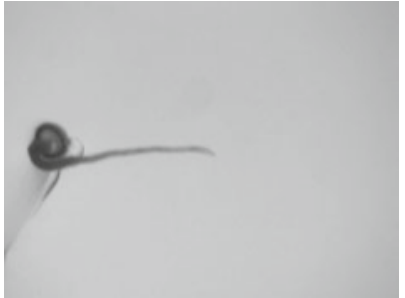

Fig. 19 Seedling with thin roots

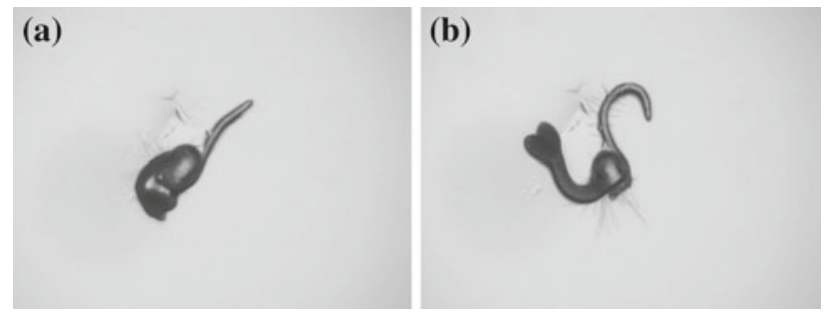

Fig. 20 Whole seedling rotates during data capture

curvature or angle measurements a challenge and so these seedlings are rejected as outliers. In some cases this causes errors in identifying the root tip and results in servoing errors. It may lead to the servoing stopping prematurely or straying resulting in a blank image, etc.

(g) Seedling shifting/rotation-the seedlings, due to the force generated by the turning of the root tip and the hypocotyl simultaneously cause the seedlings to rotate (see Fig. 20), this may cause erroneous angle measurements. In addition, the germinating seedling may shift from the original planted location of the seed. In most cases the system can still identify the root and root tip.

(h) Condensation effects-during an experiment condensation builds up on the inside of the Petri dish lid (on the face devoid of the agar growing medium). This condensation build up negatively affects the image intensity and contrast and therefore the performance of many automated thresholding techniques. This makes automated thresholding schemes to predict erroneous thresholds.

The "error conditions" listed above impact overall performance. Averaging over many data gathering sessions these data losses are approximately $5-10 \%$.

Data losses due to internal factors (system errors) In evaluating the robot system performance, we exclude the above cases, and assess only the drop in effective yield due to drawbacks in the system. The following situations arise from errors in the robot focus/servo techniques and hence are potential avenues for improved yield.

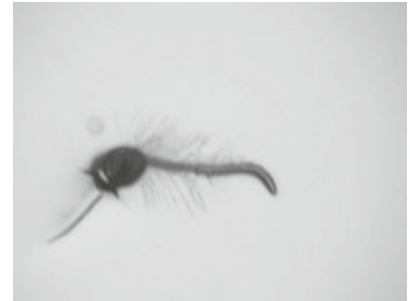

Fig. 21 Motion blur induced when operating robot at high speeds

(i) Out of focus roots- the errors in focusing are primarily due to the fact that the Petri dish and growing media are all transparent. Thus during the focus searching process the focus measure (measuring image contrast) can be affected by scratches, dust, bubbles in growing media and condensation, all effect the image contrast.

(j) Vibration blur-blur is seen when the robot speeds are set very high as the jerk generated is large during starting and stopping (shown in Fig. 21), making it necessary to have a settling time. Currently to avoid having very large settling times the gantry robot is operated at about $30 \%$ of the maximum speed.

(k) Blank images - these images result from (1) non germination of the seed causing the servoing to stray (as it cannot find the root tip) and (2) Erroneous identification of a seedling.

The effective yield is determined as the percentage of seedlings (with useful data collected) versus the available "good" (discounting those seedlings that fall into categories (a) to (h)) seedling in the cassette. This measure, only considers the mistakes performed by the system (categories (i) to (k)). As the effective yield is an adjusted measure (discounting the non system loss in data collection) it is possible to achieve $100 \%$ under ideal system performance. From the data collection sessions so far the effective yield has varied from $85 \%$ to about $95 \%$. With further improvements to the servoing and focusing system the effective yield can potentially be increased by about $5 \%$.

\subsubsection{Assessment of the root segmentation technique}

The performance of the root segmentation technique was measured by the number of successful root and root tip detections, while handling all the constraints mentioned in Sect. 4 as effectively as possible. A seedling was deemed successfully detected if the root was correctly found and the servoing positioned the root tip at the image center of the image (within a $100 \times 100$ pixel window). Table 3 provides the information on the success rate. From the table we note that the system detects the root and is successful $87.4 \%$ when a root exists. It is harder to determine the false positive, this is because 
Table 3 Overall performance in terms of effective number of seedlings found, i.e., number of useful image stacks of seedlings acquired from which phenotypic information can be extracted

\begin{tabular}{ll}
\hline Seedlings planted (Image stacks) & 3024 \\
Total failure & 633 \\
Number of seedlings for which & 289 \\
$\quad$ useful data could not be acquired & \\
due to factors not related to the & \\
$\quad$ segmentation technique & 2735 \\
Good data potentially available & 344 \\
Effective errors & 87.4 \\
Effective yield (\%)
\end{tabular}

If the system found a seedling and servo to the root tip (bringing it within the field of view) it was deemed a success

with the current frame work the region with the most rootness will be detected and in the case when no root is present, the region with the highest rootness will be used. We allow this to happen as we are not enforcing a hard threshold on the minimum rootness value that we expect for a root region.

The failures were of two kinds: (a) errors in segmentation or tracking or (b) missing seedlings. The segmentation errors were caused by incorrect identification of the root. Errors of type (b) are due to external factors, such as seeds that do not germinate, roots that grow out of the plane of the Petri dish, etc. These seedlings are excluded from the success calculations. Errors of type (a) are caused because the image quality is poor. Other reasons are a result of factors mentioned in Sect. 5.2.1.

We performed a comparison of the output of probability based segmentation technique with a standard implementation of Otsu [18] to determine the threshold. Some of the results are shown in Fig. 23. From the images in Fig. 23a we see that histogram is unimodal and thus do not expect Otsu to perform very well (as the primary assumption in the Otsu algorithm is that the image is bimodal, as with many other automated thresholding techniques). But, we see that as image quality improves Otsu performs well (e.g., row three in Fig. 23). We notice a similar scenario for the case of the Canny [2] edge detector. In the case of Canny edge detector when used on cleaner images (better quality) it is possible to obtain usable results, but the parameters will need to tuned for each image. Figures $9 \mathrm{c}$ and 22 shows the output of the edge detection, using Canny, with hand tuned input parameters (sigma of the smoothing kernel and threshold). The output degrades when using fixed values for all images and as the image quality degrades.

Section 4 introduced the use of the prior term $p(r)$. This term was obtained empirically from 300 clean images. Because this value is used as a threshold (of sorts) we tested the sensitivity of the output to it by varying the value of $p(r)$ from $0.02 \rightarrow 0.10$ with no significant change in the

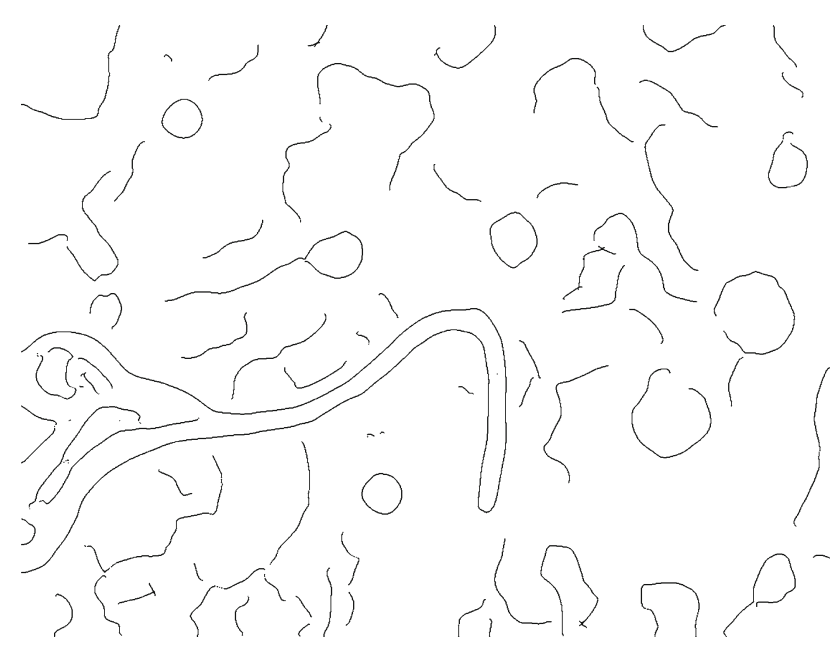

Fig. 22 Output of Canny edge detector when manually tuned for each image

segmented image, when image quality was good. But, when the image quality was very poor (i.e. completely out of focus or containing scratches, condensation etc.) the value of $p(r)$ had to kept within the range $[0.027,0.05]$. The increase in sensitivity to the value of the prior was due the shifting of the histogram towards the lower gray values, as the poor images had lower $g_{\text {avg }}=E[g]$. In addition the histograms of the poor quality images were spread out over larger ranges of gray levels especially when images were completely out of focus. The likelihood function $p(g \mid r)$, we assumed a linear model, which is represented in Eq. (3). From the equation it can be noted that highest probabilities are assigned to the darkest gray levels and the value decreases monotonically until the mean gray level of the image. All other gray levels are assigned a zero value. Ideally, a model based on the histogram information of numerous sample images of the root can be built. Other models with exponentially decaying trends were tested, but the model in Eq. (3) is simple and works equivalently well.

\subsection{Throughput comparison}

In order to demonstrate the throughput advantage that the system has over other methods of data acquisition another test was performed where 1700 seedling were imaged. The time required was 28 data gathering sessions, accounting for a yield of about $82 \%$. The data gathering session is dependent on the biology being observed, for gravitropic experiments it typically lasts for about $8 \mathrm{~h}$ and will have to be the same irrespective of the technique used for data gathering. If the same task were performed with a bank of 10 static cameras with one camera observing one seedling in one Petri dish as used in [15] it would have taken about 180 gathering sessions, even if we assume $100 \%$ yield. We assume $100 \%$ yield as 
(a)
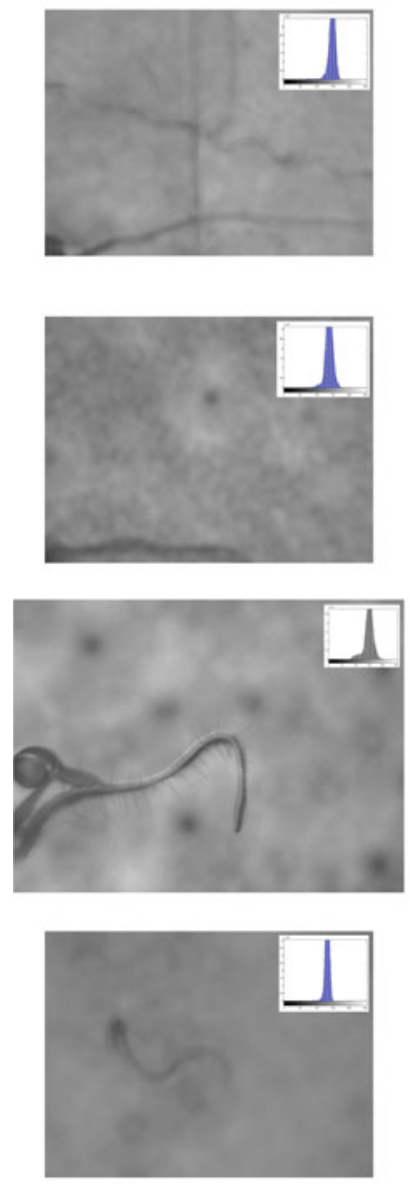

(b)
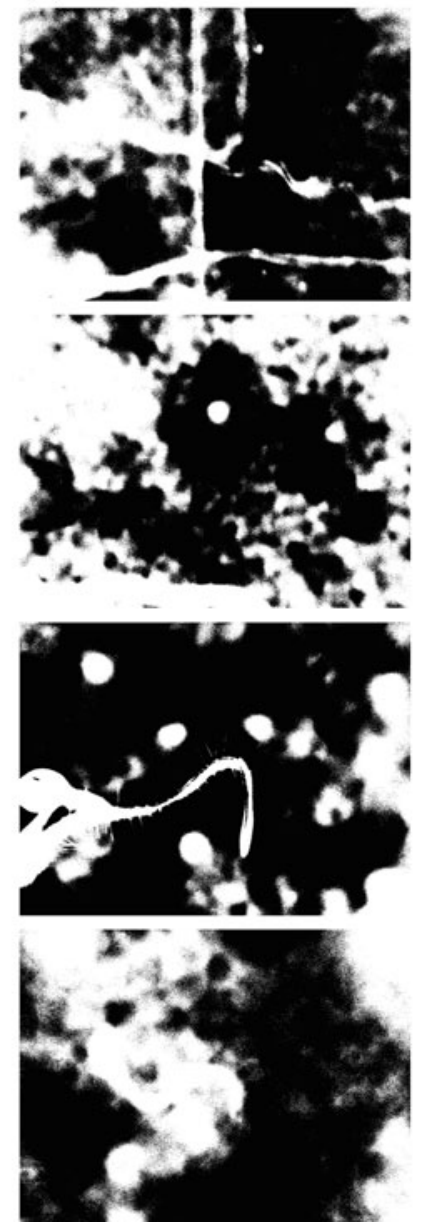

(c)
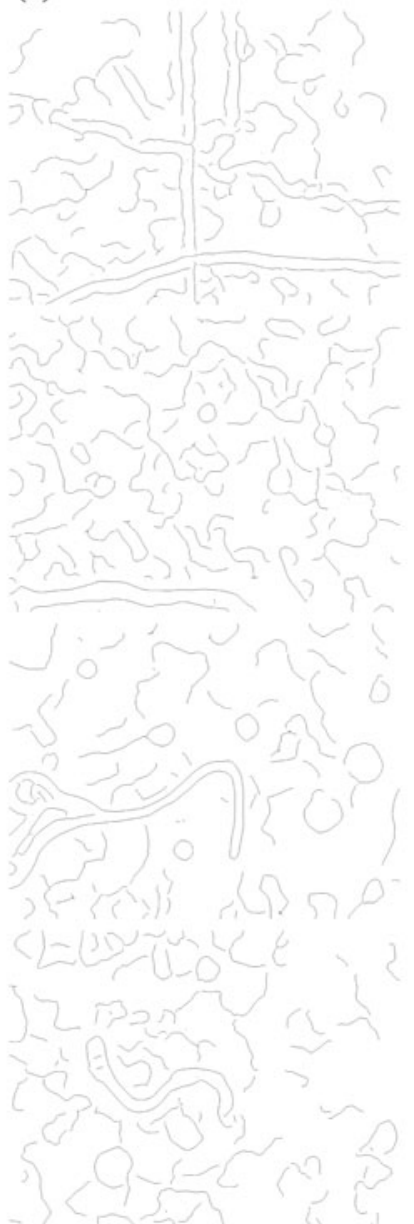

(d)
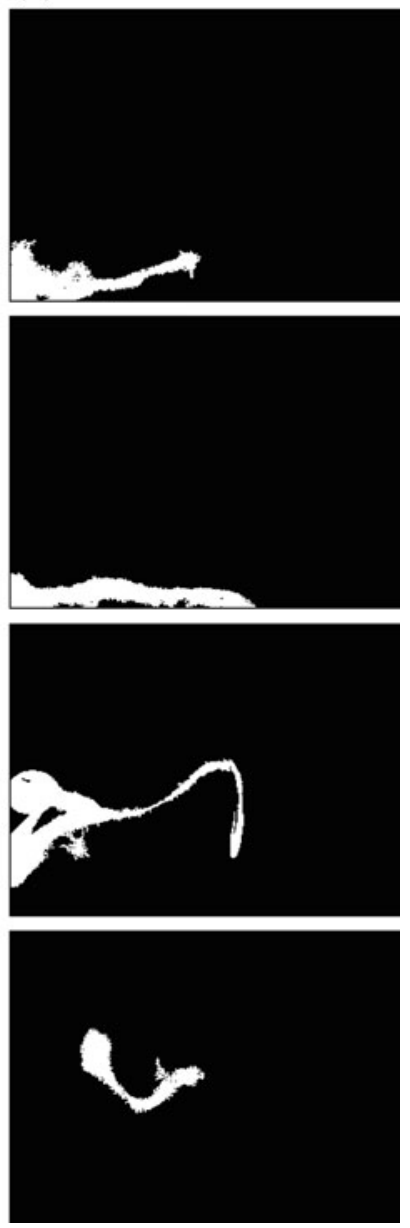

Fig. 23 The images show the difference in output between the probabilistic approach and a standard implementation of a thresholding algorithm, e.g. Otsu. a Original image captured, b output of thresholding when using Otsu algorithm to automatically estimate the threshold

no extensive studies on stationary camera systems have been performed or reported in the literature. If we assume a similar yield as we have seen in the robotic system the number of sessions required to collect the same dataset would be about 200. Further optimization of the robotic system can lead to an order of magnitude advantage with respect to data gathering time.

The number of sessions required for the stationary camera system would vary with the number of stationary camera units. But with the increase in number of camera units, cost (as can be seen from Table 4) and logistic issues arise (e.g. maintaining consistent environmental conditions over long periods of time, many months, will be a more difficult task). Additionally, the throughput of the robotic system can be further increased (potentially doubled) by planting more than one seedling close to each other such that they are within the field of view of the high magnification camera. There are

value, $\mathbf{c}$ output of Canny edge detector when manually tuned for each image (images do not have the same parameter values), and $\mathbf{d}$ output from the probabilistic segmentation technique

complications that will arise and further work is currently required to make this scenario work well.

\subsection{Setup analysis}

In addition to the way the system operates and performs, we carried out an analysis of the cost to setup the system and how these costs would change with as the acquisition system scaled. The costs are representative and were only used to determine if the setup cost would be high or low in each case. Table 4 shows the cost per seedling for the entire imaging equipment along with some of the pros and cons of each type.

The single camera system referred to here is similar to those used in [15]. Table 4 gives an approximate (order of magnitude) setup comparison for the high throughput options. When scaling the size of the study the robotic system 
Table 4 Robotic system versus manual and semi-automated systems

\begin{tabular}{|c|c|c|}
\hline System & Advantage & Disadvantage \\
\hline $\begin{array}{l}\text { Manual } \\
\text { (1 camera, } \\
1 \text { seedling) }\end{array}$ & $\begin{array}{l}\text { Can provide high } \\
\text { image and time } \\
\text { resolution. } \\
\text { With } 1 \text { to } 5 \\
\text { cameras, it is easy } \\
\text { to mange planting } \\
\text { and scheduling. }\end{array}$ & $\begin{array}{l}\text { Very low } \\
\text { throughput. } \\
\text { Cost of setting up } \\
\text { up scales linearly } \\
\text { with increase in } \\
\text { number of cameras. } \\
\text { Complex logistics } \\
\text { with many cameras } \\
\text { Dataset collection } \\
\text { is very long drawn } \\
\text { (many months). }\end{array}$ \\
\hline $\begin{array}{l}\text { Semi } \\
\text { Automated } \\
\text { (1 camera, } \\
\text { many } \\
\text { seedlings) }\end{array}$ & $\begin{array}{l}\text { Higher throughput } \\
\text { than single camera } \\
\text { system. } \\
\text { Lower setting up } \\
\text { cost per seedling. } \\
\text { High time } \\
\text { resolution. }\end{array}$ & $\begin{array}{l}\text { Lower image } \\
\text { resolution. } \\
\text { Long dataset } \\
\text { collection time. } \\
\text { Prone to logistical } \\
\text { problems similar } \\
\text { to Manual } \\
\text { collection. }\end{array}$ \\
\hline $\begin{array}{l}\text { Semi } \\
\text { Automated } \\
\text { (10 cameras, } \\
10 \text { seedlings) }\end{array}$ & $\begin{array}{l}\text { Relatively higher } \\
\text { throughput than } \\
\text { Manual. } \\
\text { High image and } \\
\text { time resolution. }\end{array}$ & $\begin{array}{l}\text { Suffers from all } \\
\text { the disadvantages } \\
\text { as the single } \\
\text { camera manual } \\
\text { collection. }\end{array}$ \\
\hline Robotic & $\begin{array}{l}\text { Very high } \\
\text { throughput and } \\
\text { image resolution. } \\
\text { Much shorter } \\
\text { collection time } \\
\text { Lowest, per } \\
\text { seedling set up } \\
\text { cost and space } \\
\text { occupied. } \\
\text { Organizing and } \\
\text { scheduling is } \\
\text { automated. } \\
\text { Easier to maintain } \\
\text { experimental } \\
\text { consistency. } \\
\text { Easily adaptable } \\
\text { for various seed } \\
\text { types and } \\
\text { experimental } \\
\text { conditions. }\end{array}$ & $\begin{array}{l}\text { Although costs } \\
\text { amortize very } \\
\text { quickly, initial } \\
\text { over all cost } \\
\text { is high. } \\
\text { Most suited for } \\
\text { large scale data } \\
\text { collection. }\end{array}$ \\
\hline
\end{tabular}

has a much lower amortized setup cost. In addition, these costs amortize much faster for the robotic system due to the high throughput in data collection.

\subsection{System bottlenecks}

From the analysis of the system in the earlier part of this section we can identify the most critical parameters of the system that can effect the throughput. The bottleneck can be identified by analyzing the temporal sampling ability. This is the shortest time between two observations of a seedling. This is determined by the term $t_{\text {gatherall }}$ given by Eq. (12). Typically, this can range from a few hours to about 5 min depending on the nature of the phenotype. In the case of a manual data gathering process a few (typically 5-10) seedlings are imaged every hour or two. With the semi-automated scenario one camera focused on one seedling can gather image data of the region of interest at a resolution of about $2 \mathrm{~min}$ or less. With the robotic system at present, with the cassette loaded with 72 seedlings, the finest resolution is about $3.5 \mathrm{~min}$ (with the exception of $t_{\mathrm{fp}}$, first pass time). The major factors that affect the temporal resolution are: (a) Size and speed of system and (b) Number of seedlings.

From Eq. (12) it can be seen that $t_{\text {gath }}$ increases linearly with increase in $N$, number of seedlings. Also, owing to the accuracy and speed of the system all values in $t_{\text {gath }}$ are small, which when improved will lead to a total reduction of $t_{\text {gatherall }}$ by $5 \%$. Thus, this is not a critical bottleneck.

In the case of $t_{\mathrm{fp}}$, as discussed in Sect. 5.1, $t_{\text {servo }}$ and $t_{\text {focus }}$ constitutes the largest share of the time. Using Eqs. (14) through 15 it can be seen that maximum gain in $t_{\mathrm{fp}}$ can be obtained by reducing $t_{\text {servo }}$ and $t_{\text {focus }}$. This will allow for an increase in throughput when collecting data for biological processes with earlier onset times $\left(t_{\text {onset }}\right.$, discussed in Sect. 5.1). $t_{\text {servo }}$, time taken to servo to the root tip, is dependent on the length of the seedling and varies between 1 and $5 \mathrm{~s}$, while $t_{\text {focus }}$, time to focus on a given root is typically between 5 and 16 seconds. Thus reducing focusing time by 5 seconds can reduce $t_{\mathrm{fp}}$ by about $25 \%$. Thus establishing that the critical bottleneck for $t_{\mathrm{fp}}$ is currently the focusing process and further research is required to provide faster on-line focusing technique.

\section{Discussions and future work}

A system for high throughput phenotyping has been described in detail and tested with the specific model plant, Arabidopsis thaliana, for root gravitropic experiments. The performance of the system was analyzed and its capabilities, advantages and shortcomings have been presented.

Currently, the system is used to track the properties of the root (growth, curvature and tip angle) of Arabidopsis seedlings. Our future work would be to extend the capabilities of the system to monitor the whole seedling, enabling phenotypic quantification of other structures and processes, such as shoot development. This will also open up the possibility of 
correlating all the properties and measurements. In addition, we would like to explore ways to adapt the system to monitor other plant species (like maize, rice etc.)

Open Access This article is distributed under the terms of the Creative Commons Attribution License which permits any use, distribution, and reproduction in any medium, provided the original author(s) and the source are credited.

\section{References}

1. Basu, P., Pal, J., Lynch, J., Brown, K.: A novel image-analysis technique for kinematic study of growth and curvature. Plant Physiol. 145, 305-316 (2007)

2. Canny, J.F.: A computational approach to edge detection. IEEE Trans. Pattern Anal. Mach. Intell. 8(6), 679-698 (1986)

3. Chaumette, F., Hutchinson, S.: Visual servo control. Part I: basic approaches. IEEE Robot. Autom. Mag. 13(4), 82-90 (2006)

4. Chaumette, F., Hutchinson, S.: Visual servo control. Part II: advanced approaches. IEEE Robot. Autom. Mag. 14(1), 109118 (2007)

5. Corke, P., Hutchinson, S.: A new hybrid image-based visual servo control scheme. In: Proceedings of the 39th IEEE Conference on Decision and control (2000)

6. Corke, P., Hutchinson, S.: A new partitioned approach to imagebased visual servo control. IEEE Trans. Robot. Autom. 17(4), 507515 (2001)

7. French, A., Ubeda-Tomas, S., Holman, T., Bennett, M., Pridmore, T.: High-throughput quantification of root growth using a novel image-analysis tool. Plant Physiol. 150, 1784-1795 (2009)

8. Groen, F.C., Young, I.T., Ligthart, G.: A comparison of different focus functions for use in autofocus algorithms. Cytometry, pp. 623-691 (1985)

9. Hutchinson, S.A., Hager, G.D., Corke, P.I.: A tutorial on visual servo control. IEEE Trans. Robot. Autom. 12(5), 651-670 (1996). http://citeseer.ist.psu.edu/hutchinson96tutorial.html

10. Ishikawa, H., Evans, M.: Novel software for analysis of gravitropism: comparative response patters of Arabidopsis wide-type and axr1 seedlings. Plant Cell Environ. 20, 919-928 (1997)

11. Jaffe, M., Wakwfield, A., Telewski, F., Gulley, E., Biro, R.: Computer-assisted image analysis of plant growth, thigmomorphogenesis and gravitropism. Plant Physiol. 77, 722-730 (1985)

12. Kristan, M., Pers, J., Perse, M., Kovacic, S.: A bayes spectral entropy based measure of camera focus using a discrete cosine transform. Pattern Recognit. Lett. 27, 1431-1439 (2006)

13. Krotkov, E.: Focusing. IJCV 1, 223-237 (1987)

14. LemnaTec: http://www.lemnatec.com (1998)

15. Miller, N., Parks, B., Spalding, E.: Computer-vision analysis of seedling responses to light and gravity. Plant J. 52(2), 374381 (2007)

16. Mullen, J., Wolverton, C., Ishikawa, H., Evans, M.: Kinetics of constant gravitropic stimulus responses in Arabidopsis roots using a feedback system. Plant Physiol. 123, 665-670 (2000)

17. Nathaniel, N.K.C., Neow, P.A., M.H.A. Jr.: Practical issues in pixelbased autofocusing for machine vision. In: ICRA, p. 2791 (2001)

18. Otsu, N.: A threshold selection method from gray-level histograms. IEEE Trans. Syst. Man. Cyber. 9, 6266 (1979)

19. Song, Y., Sun, L.: A new auto focusing algorithm for optical microscopebased automated system. In: ICARCV (2006)

20. Sun, Y., Duthaler, S., Nelson, B.: Autofocusing algorithm selection in computer microscopy. In: Proceedings of the IEEE Conference on IROS (2005)
21. Tahri, O., Chaumette, F.: Image moments: generic descriptors for decoupled image-based visual servoing. In: Proceedings of the IEEE Conference on Robotics and Automation, pp. 1861-1867 (2004)

22. Tahri, O., Chaumette, F.: Complex objects pose estimation based on image moment invariants. In: Proceedings of the IEEE Conference on Robots and Automation, pp. 436-441 (2005)

23. Walter, A., Spies, H., Terjung, S., Kusters, R., Kirchgebner, N., Schurr, U.: Spatio-temporal dynamics of expansion growth in roots: automatic quantification of diurnal course and temperature response by digital image sequence processing. J. Exp. Biol. 53, 689-698 (2002)

24. Wang, L., Uilecan, I., Assadi, A., Kozmik, C., Spalding, E.: Hypotrace image analysis software for measuring hypocotyl growth and shape demonstrated on Arabidopsis seedlings undergoing photomorphogenesis. Plant Physiol. 149, 1632-1637 (2009)

25. Yap, P., Raveendran, P.: Image focus measure based on Chebyshev moments. IEE Proc.-Vis. Image Signal Process. 151(2), 128 (2004)

\section{Author Biographies}

Ram Subramanian received his Ph.D. degree from the Department of Mechanical Engineering at the University of Wisconsin-Madison in 2012 where he worked in the robotics and intelligent systems laboratory. Dr. Subramanian received his bachelors' degree in electrical engineering from the University of Madras in 2001 and his master's degrees in biomedical engineering and mechanical engineering at the University of Wisconsin-Madison. Dr. Subramanian's research interests include image-based robot control and image-based metrology with applications ranging from medical imaging to plant phenotyping.

Edgar P. Spalding received his Ph.D. degree from the Department of Biology at Pennsylvania State University in 1990 under the supervision of Daniel Cosgrove. He was a postdoctoral fellow at Yale University from 1990 to 1994. He joined the University of Wisconsin's Department of Botany faculty in 1994. Professor Spalding's research interests are in the transport of ions including auxin, photomorphogenesis and phenotype frameworks. He was awarded the NSF early Faculty Career Development Award from 1998 to 2002, Vilas Research Associate 2005 to 2007 and Hamel Family Faculty Fellow 2008-2013. Professor Spalding is a fellow of the American Society of Plant Biologists and has served the society in various capacities including Secretary and Board of Trustees.

Nicola J. Ferrier (IEEE Member since 1991) received her Ph.D. degree from the Division of Applied Sciences at Harvard University in 1992 where she worked in the Laboratory for Robotics, Decision and Control. She was a Junior Research Fellow at the University of Oxford from 1992 to 1994 in the Robotics Research Group and a post-doctoral fellow at Harvard University in 1995. She joined the University of Wisconsin's Mechanical Engineering faculty in 1996. Professor Ferrier's research interests are in the use of computer vision/imagery to control robots, machinery, and devices, with applications as diverse as medical systems, manufacturing, and navigation. The use of visual feedback in the control of a robot end-effector position is advantageous when precise fixturing is impractical or impossible. Her work has been applied to visual monitoring of polymer processing, visual control of crystal formation processes, and visual monitoring of nano-scale assembly processes. Professor Ferrier received the University of Wisconsin Vilas Associate Award for research in 1999 and the National Science Foundation CAREER Award in 1997. 